\title{
REPARACIÓN ÓPTIMA DE ESCUELAS CON MUROS DE MAMPOSTERÍA Y MARCOS DE CONCRETO CON DAÑOS SÍSMICOS BASADA EN CONFIABILIDAD
}

\author{
José Luis García Manjarrez ${ }^{(1)}$ y David De León Escobedo ${ }^{(1)}$
}

\begin{abstract}
RESUMEN
El presente trabajo utiliza las técnicas de confiabilidad estructural para identificar la reparación óptima de estructuras con un sistema dual, concreto, y muros de mampostería, así como los costos y el ciclo de vida, para generar recomendaciones prácticas sobre el tipo de refuerzo para el estado límite de resistencia y el de servicio. Se incorporan los estados límite de resistencia y de servicio, y se analiza el comportamiento combinado del marco y los muros. Se identifican los posibles modos de falla desde la capacidad de flexión y corte hasta la deriva entre pisos en dos escuelas ubicada en Yautepec y Tlatenchi, en el Estado de Morelos. Las escuelas sufrieron daños considerables en los muros, afectando su nivel de seguridad después del sismo ocurrido el 19 de septiembre del 2017. Mediante la aplicación de la confiabilidad estructural y los conceptos de costos esperados en el ciclo de vida, se propone una formulación para generar niveles óptimos de actualización de seguridad para reparar escuelas dañadas por sismos. Se analizan los modelos con un software comercial (PERFORM 3D V7) y se obtienen las respuestas máximas para las escuelas mencionadas. Se utilizan coeficientes de variación de carga y resistencia reportados en la literatura, para obtener la probabilidad de falla de la escuela. La probabilidad de falla de una estructura para cada estado límite, se evalúa bajo las condiciones dañadas de la estructura, luego, sucesivamente, se proponen varios tipos de reforzamiento, y se calculan las probabilidades de falla correspondientes y el costo esperado en el ciclo de vida. Se analizan 3 alternativas de reparación, la introducción de: concreto reforzado, ángulos y soleras de acero y muros de concreto reforzado. La reparación óptima será aquella para la que se cumpla la del costo mínimo esperado en el ciclo de vida. Las complejidades del tema brindan la oportunidad para realizar trabajos multidisciplinarios, los cuales pueden ser una herramienta muy favorable para generar recomendaciones prácticas para ingenieros a cargo de reparaciones de estructuras.
\end{abstract}

Palabras clave: confiabilidad; reparación; mampostería; ciclo de vida; reforzamiento; consecuencias de fallas

\footnotetext{
Artículo recibido el 14 de mayo de 2021 y aprobado para su publicación el 30 de diciembre de 2021. Se aceptarán comentarios y/o discusiones hasta cinco meses después de su publicación.

(1) Facultad de Ingeniería, Universidad Autónoma del Estado de México, Ciudad Universitaria, Toluca Estado de México, México, j1tae-music@ hotmail.com ; daviddeleonescobedo@yahoo.com.mx
}

DOI: $10.18867 /$ ris. 106.590 


\title{
OPTIMAL RELIABILITY BASED REPAIR FOR SCHOOLS WITH MASONRY WALLS AND CONCRETE FRAMES THAT SUFFERED SEISMIC DAMAGE
}

\begin{abstract}
This investigation uses structural reliability techniques for identifying optimal repair option of a structures with a dual system, concrete, and masonry walls. Additionally, it analyzes costs and service life to generate practical recommendations on the type of reinforcement for the limit state of resistance and service. Resistance and service limit states are included, and the combined performance of the frames and masonry walls are analyzed. Possible failure modes are identified, from bending and shear to drift between two floors in two different schools located in Yautepec and Tlatenchi, in the State of Morelos. The schools suffered serious damages in the walls that affected their safety levels after the earthquake of September 19 of 2017. Through the application of structural reliability and the concepts of the expected life cycle costs, a formulation is proposed to develop optimal reliability upgrading recommendations to repair schools damaged by earthquakes. The models are analyzed with a commercial software (PERFORM 3D V7) to obtain the maximum responses for the aforementioned schools. The coefficient of variation of load and resistance reported in the literature, are used to obtain the school's failure probability. The failure probability of a structure for each limit condition, is evaluated under the damaged conditions of the structure, and, successively, different types of retrofits are proposed. Then the corresponding failure probabilities and the expected cost in the life cycle are calculated. Three repair alternatives are analyzed: reinforced concrete, steel flat strips and angles, and concrete reinforced walls. The optimal repair will be the one that renders the expected minimum life cycle cost. The complexities of the subject provide the opportunity to conduct multidisciplinary investigations that can be a convenient tool to generate practical recommendations for engineers in charge of structural repairs.
\end{abstract}

Keywords: reliability; repair; masonry; life cycle; reinforcement; failure consequences

\section{INTRODUCCIÓN}

Tras los sismos del 7 y 19 de septiembre de 2017, se registraron afectaciones en 19,194 escuelas, de las cuales 210 reportaron daños graves o requerían reconstrucción total. Mientras tanto, en otras 6,970 escuelas hubo daños que fueron clasificados como de moderados a severos, y 12,014 escuelas con daño menor, que requerían reparar acabados, pero no demandaron hacer reparaciones a nivel estructural (Instituto Belisario Domínguez, Senado de la República, 2017).

Los daños producidos por estos sismos aceleraron en México los estudios para dotar de protección sísmica adecuada a escuelas y otras construcciones (Sánchez e Islas, 2017; García y otros, 2018; Alcocer y otros, 2019; INIFED, 2021). El peligro sísmico de la Ciudad de México ha sido un motivo continuo de atención (Ordaz y Reyes, 1999; Reinoso, 2007; Cruz, 2013; Tena-Colunga y otros, 2021). Por ello es conveniente determinar el nivel de peligro y riesgo sísmico que presentan las estructuras en cada zona, en especial las destinadas a escuelas, pero, sobre todo, generar propuestas de reforzamiento que permitan tener la seguridad adecuada ante eventos sísmicos de gran magnitud. 
Tabla 1. Daños en escuelas por el terremoto del 19 de septiembre de 2017 en México (Instituto Belisario Domínguez, 2017)

\begin{tabular}{lcccc}
\hline \multicolumn{1}{c}{ Estado } & No. de escuelas dañadas & $\begin{array}{c}\text { Nivel 3. } \\
\text { Menor }\end{array}$ & $\begin{array}{c}\text { Nivel 2. } \\
\text { Moderado }\end{array}$ & Nivel 1. Grave \\
\hline 1) Estado de México & 4,909 & 2,127 & 2,747 & 35 \\
2) Chiapas & 3,067 & 2,147 & 916 & 4 \\
3) Oaxaca & 3,001 & 1,879 & 1,094 & 28 \\
4) Puebla & 2,272 & 1,610 & 585 & 77 \\
5) Ciudad de México & 2,006 & 1,169 & 828 & 9 \\
6) Morelos & 1,341 & 904 & 396 & 41 \\
7) Tlaxcala & 1,013 & 814 & 198 & 1 \\
8) Michoacán & 570 & 559 & 11 & N/A \\
9) Guerrero & 550 & 419 & 119 & 12 \\
10) Hidalgo & 413 & 368 & 42 & 3 \\
11) Veracruz & 52 & 18 & 34 & N/A \\
$\quad$ Total & 19,194 & 1,2014 & 6,970 & 210 \\
\hline
\end{tabular}

Diversos autores destacan los factores comunes que hacen que las estructuras sean vulnerables, como los daños acumulados de las construcciones, que no reciben reparación antes del próximo terremoto, o que muestran otro tipo de daños (CENAPRED, 2001; Cruz, 2002; Tolentino, 2009). En un estudio reciente sobre vulnerabilidad de escuelas en países en desarrollo, se encontró que en muchos casos las escuelas se construyen con materiales no dúctiles, con salas muy amplias y con grandes ventanas, como por ejemplo en Haití, Nepal, Mongolia e Indonesia. Estos son factores que contribuyen a la vulnerabilidad sísmica, como se ha documentado en escuelas de países en desarrollo (Gilani, 2018).

En el tema de las técnicas para la reparación postsísmica en estructuras, en 2006, Federal Emergency Management Agency (FEMA 547, 2006) propuso una serie de técnicas para la reparación sísmica de edificios. Se presentó un catálogo de técnicas de reparación de edificios sencillos bajo los auspicios de las Naciones Unidas, World Seismic Safety Initiative. Este libro incluye descripciones de daño estructural y no estructural, y ofrece ayudas gráficas para hacer reparaciones en condiciones de auto construcción (Teddy y Asociados, 2010). En México, existe un manual en que se puede observar el nivel de daño que tuvo un elemento estructural dependiendo de los anchos de grietas, así se obtiene la pérdida de resistencia de un elemento estructural, para estimar la degradación de resistencia de un elemento dañado, Centro Nacional de Prevención de Desastres (CENAPRED, 2001). Además, el Instituto Nacional de la Infraestructura Física Educativa apoyó un proyecto de investigación para generar guías para la evaluación post sísmica de la infraestructura (INIFED, 2021), para encamisar con concreto reforzado y ángulos y soleras de acero. Por otra parte, existen manuales que tiene cobertura en todo el territorio mexicano, el de la Comisión Federal de Electricidad (CFE, 2015), y las Normas Técnicas Complementarias de la Ciudad de México (Gobierno de la Ciudad de México, 2017), contribuyen a aportar guías técnicas, aunque en su mayor parte se orientan al diseño y no a la reparación de estructuras. Recientemente, se han hecho propuestas para reparar instalaciones para escuelas (Alcocer y otros, 2020; Mansor y Siang, 2019) considerando las consecuencias de fallas potenciales (De León e Ismael, 2020). Pese a estos avances, los ingenieros de la práctica se apoyan en su experiencia y criterios subjetivos, para determinar el tipo de reparación y el nivel de seguridad que debe buscarse para la construcción reparada. La ausencia de guías sistemáticas que orienten al ingeniero hacia el tipo y nivel de reparación que optimiza la relación costo/beneficio, revela nichos de oportunidad en este tema.

Dado que aún no se han implementado estrategias óptimas, el presente documento tiene como objetivo contribuir en este campo, mostrando un enfoque basado en la confiabilidad estructural para identificar la alternativa óptima de la reparación, entre tres opciones propuestas, desde el punto de vista del 
costo mínimo esperado en el ciclo de vida. Las alternativas propuestas son el encamisado de concreto, el encamisado con ángulos y soleras de acero y la introducción de muros de concreto.

En un trabajo similar al aquí propuesto se presentó un análisis costo/beneficio para escuelas públicas en México (Jaimes y Niño, 2017). En el presente trabajo se hace énfasis en escuelas con un sistema estructural de marcos de concreto y muros. Vale la pena comentar que la mayoría de las escuelas de todo el país utilizan un sistema estructural dual: marco de concreto o acero y muros de mampostería.

\section{METODOLOGÍA}

La siguiente metodología se usa para obtener la mejor alternativa de reparación considerando la relación Costo- Beneficio, la cual se ilustra en el diagrama de bloques de la figura 1.

Se utilizó un registro sísmico del sismo del 19 septiembre del 2017 para ambas escuelas, con clave YAIG, con una duración del registro de $900 \mathrm{~s}$, con una distancia de la estación a las escuelas de 31.5 y 24.72 kilómetros, producto de las labores de instrumentación y procesamiento de la unidad de instrumentación Sísmica del Instituto de Ingeniería de la UNAM (II-UNAM, 2021).

Ante la duda de los refuerzos de acero en las secciones para las escuelas estudiadas, ya que no se encontraron en los planos, se realizó un proceso de calibración: se utilizaron los daños observados y los registros cercanos a cada escuela, para aproximar las propiedades mecánicas de las escuelas. Este proceso de calibración consistió en lo siguiente. En general, se deben obtener los planos estructurales de la estructura, tal como fue construida para el modelado, para este trabajo se hizo uso de la calibración del modelo en función de los daños observados ya que los modelos que se estudiaron se demolieron en el 2017, y no era posible investigar en campo las secciones transversales. De los planos se tomaron las dimensiones de las secciones originales de la estructura y se propuso, de inicio, un área de acero mínima, ya que es la única variable desconocida. Después de realizar el análisis estructural, se identifican los elementos con mayor cociente (demanda/ capacidad), se obtiene el índice de confiabilidad $\beta$, y dependiendo del daño observado 0-1 severo (falla total del elemento estructural), 1-2 moderado (reparación estructural), 2-3 menor (reparación no estructural o estética) (CENAPRED, 2001), se va aumentando la cantidad de acero de refuerzo del elemento, hasta que se ajuste con el índice de confiabilidad $\beta$ asociado de manera intuitiva al daño observado según la escala mencionada. Para aplicar la metodología de la figura 1, en general, para estructuras existentes sería necesario realizar los estudios adecuados para conocer la resistencia del concreto de los diferentes elementos estructurales mediante el uso del esclerómetro y la extracción de núcleos de concreto, además se tendrá que realizar un escaneo de acero de refuerzo para conocer el diámetro, cantidad y separación del acero longitudinal y transversal. Por las razones ya mencionadas, en estos casos no fue posible realizar estos estudios. Las propuestas de reparación se evaluaron, considerando los modelos reforzados, calculando el índice de confiabilidad global y el costo esperado en el ciclo de vida. La alternativa óptima es la que representa el menos coto esperado en el ciclo de vida.

El aspecto del daño acumulado por varios sismos y la degradación de la estructura (Tolentino, Ruiz y Torres, 2012) no se incluye en el presente trabajo. Las técnicas de reparación a considerar son: encamisado de concreto, encamisado de acero con ángulos y soleras y muros de concreto. Cada modelo de escuela reparada es sometida a los espectros sísmicos correspondientes a la ubicación, con el fin de representar los posibles terremotos futuros y se selecciona la alternativa óptima con respecto al costo esperado del ciclo de vida, el cual incluye el costo de las consecuencias de fallas. 
Espectro de respuesta y espectro de diseño del sitio

$\sqrt{5}$

Análisis y diseño estructural

$\sqrt{3}$

Calibración de la resistencia de elementos estructurales según daño observado, a partir de un análisis no lineal.

U3

Resistencia de elementos, columnas (flexo-compresión), trabes (flexión, cortante).

$\sqrt{2}$

Acciones actuantes en los elementos estructurales, análisis no lineal.

U

Probabilidad de falla e índice de confiabilidad $\beta$ de los miembros críticos.

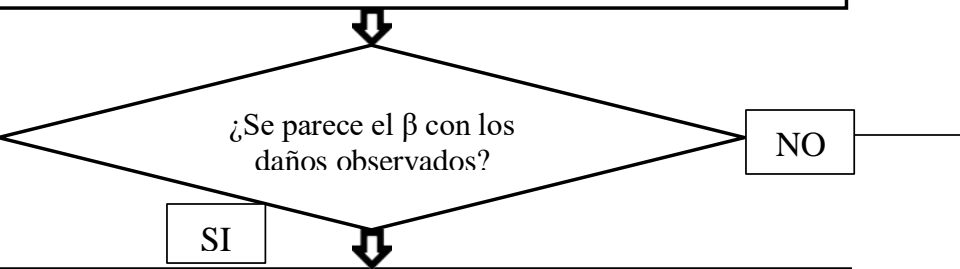

Propuesta de reparación de elementos con las mayores probabilidades de falla.

J

Nueva resistencia de elementos reforzados, columnas (flexocompresión), trabes (flexión, cortante).

U

Acciones actuantes en los elementos estructurales, análisis no lineal.

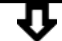

Probabilidad de falla e índice de confiabilidad $\beta$ de los elementos reforzados.

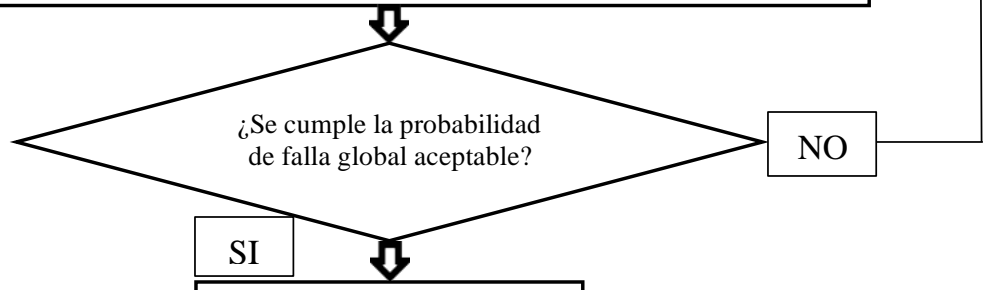

Costo de la opción

Obtener la relación costo-beneficio $(\mathrm{C} / \mathrm{B})$ para

cada opción

15

Identificar la mejor opción

Figura 1. Proceso esquemático de la formulación propuesta 


\section{Cálculo de la probabilidad de falla}

El cálculo de las probabilidades de falla de los elementos con sus propiedades originales (sin considerar el daño acumulado en las escuelas debido a sismos anteriores) se realiza a través de simulaciones de Monte Carlo que se basa en los valores medios de las respuestas máximas y los coeficientes de variación tomados de la literatura. El índice de confiabilidad $\beta$ se usa de modo aproximado, aun cuando las variables no sean Gaussianas, como es el caso en este trabajo. El índice de confiabilidad $\beta$ óptimo para escuelas, al igual que para construcciones muy importantes, esta entre 3.8 y 4.3 (The Eurocode, 2002) dependiendo del costo de las consecuencias de falla, aquí, las demandas y las resistencias se consideran como variables aleatorias log normales.

Para las trabes, se sigue una formula cerrada, ecuación 1, para el índice de confiabilidad de vigas. Para las columnas, se aplican procesos MCS (simulación de Monte Carlo) para obtener las probabilidades de falla originales y reacondicionadas. La incertidumbre se considera en la demanda sísmica y las resistencias estructurales y la actuación y resistencia se consideran lognormales. La probabilidad de falla, por ejemplo, para la flexión se calcula a través de ecuación 1. El índice de confiabilidad $\beta$ se expresa en términos de la mediana del momento actuante, $\widetilde{M_{a}}$, y del momento resistente $\widetilde{M_{r}}$, y los coeficientes de variación para momento resistente $C V_{M r}=0.15$ y para momento actuante $C V_{M a}=0.3$, los coeficientes de variación para la fuerza cortante actuante $C V_{a}=0.3$ y fuerza cortante resistente $C V_{l}=0.1$, respectivamente (De León y Guadarrama, 2020).

$$
\beta=\frac{\ln \left(\widetilde{M_{a}}\right)-\ln \left(\widetilde{M}_{r}\right)}{\sqrt{C V_{a}^{2}+C V_{r}^{2}}}
$$

En el cual $a=$ actuante, $r=$ resistente; $M$ es el momento. Las medianas de los actuantes y resistentes son:

$$
\begin{aligned}
& \bar{M}_{a}=\frac{E\left(M_{a}\right)}{\sqrt{1+C V_{M_{a}}^{2}}} \\
& \bar{M}_{r}=\frac{E\left(M_{r}\right)}{\sqrt{1+C V_{M_{r}}^{2}}}
\end{aligned}
$$

donde los valores medios de momentos actuantes y resistentes, $E\left(M_{a}\right)$ y $E\left(M_{r}\right)$, respectivamente, se toman como las respuestas y resistencias en flexión para la trabe en cuestión.

El índice de confiabilidad $\beta$, está relacionado con la probabilidad de falla a través de la distribución de Gauss acumulada $\Phi(\cdot)$ (Nowak y Collins, 2012). Cuando las variables no son normales, se aproxima la probabilidad de falla mediante:

$$
P_{f_{i}} \approx 1-\Phi\left(\beta_{i}\right)
$$

La probabilidad de falla de una estructura se considera como la falla del marco crítico, y la probabilidad de falla del marco se define aquí como la probabilidad de que un efecto de carga o efecto de combinación de carga exceda la resistencia de los miembros estructurales críticos. 
Cuando se identifican los miembros críticos, la probabilidad de falla del marco puede expresarse como el producto de la probabilidad condicional de falla del marco, dada la probabilidad de falla de los miembros críticos, asumiendo que estos eventos son independientes.

$$
\begin{aligned}
P_{f} & =P\left(F_{1} \cup F_{2} \cup F_{3}\right)= \\
& =P\left(F_{1}\right)+P\left(F_{2}\right)+P\left(F_{3}\right)-P\left(F_{1} \cap F_{2}\right)-P\left(F_{2} \cap F_{3}\right)-P\left(F_{1} \cap F_{3}\right)+P\left(F_{1} \cap F_{2} \cap F_{3}\right)
\end{aligned}
$$

donde $F_{1} \cap F_{2} \cap \ldots F_{n}$ constituye el fallo del conjunto " $s$ " de $n$ miembros, que implican el fallo del marco global.

$$
\begin{aligned}
& P\left(F_{1} \cap F_{2}\right)=P\left(F_{1}\right) P\left(F_{2}\right) \\
& P\left(F_{1} \cap F_{3}\right)=P\left(F_{1}\right) P\left(F_{3}\right) \\
& P\left(F_{2} \cap F_{3}\right)=P\left(F_{2}\right) P\left(F_{3}\right) \\
& P\left(F_{1} \cap F_{2} \cap F_{3}\right)=P\left(F_{1}\right) P\left(F_{2}\right) P\left(F_{3}\right)
\end{aligned}
$$

\section{Cálculo de costos esperados}

El costo esperado del ciclo de vida se expresa en función del costo de reparación, $C_{r}$, y el valor actual de los costos futuros esperados, $E\left(C_{F}\right)$, (Ang y De León, 1997).

$$
E\left(C_{l}\right)=C_{r}+E\left(C_{F}\right)
$$

Los costos futuros previstos $E\left(C_{F}\right)$ se expresan como:

$$
E\left(C_{F}\right)=P V F\left(C_{F}\right) P_{f}
$$

donde $P F V$ es el factor de valor presente,

$$
P V F=(1-\exp (-r T)) / r
$$

$r$ es la tasa de interés anual neta y $T$ es la vida útil de la escuela. Además, los componentes del costo futuro $\left(C_{F}\right)$ son los costos de las pérdidas por falla o colapso de la escuela, (fatalidades, instalaciones y muebles, costo de la estructura, costo de traslado de funciones escolares a otro lugar, etc.) (De León y Ang, 1994).

$$
C_{F}=C_{r}+C_{c}+C_{e}+C_{\text {in }}+C_{f}
$$

donde:

$C_{r}=$ Costo de reparación o reemplazo. Este costo se calcula generando un catálogo de conceptos, para la reparación, reconstrucción o reforzamiento estructural y posteriormente, se realiza un análisis de precios unitarios.

$C_{c}=$ Pérdida de contenidos. La estimación de la pérdida de contenido se realiza cuantificando los muebles, equipos de cómputo, laboratorios que se necesitan reemplazar. Estos costos se toman de proveedores de las escuelas mexicanas, en la zona de cada escuela, a precios actuales (Mlego, 2021).

$C_{e}=$ Pérdida económica debido a la interrupción del servicio. Se genera considerando los costos que son consecuencia de trasladar las labores escolares a un sitio temporal, durante el tiempo de reconstrucción o reemplazo y es el costo de renta de una instalación de similar tamaño; se considera un periodo de un año. 
$C_{i n}=$ Costo de las lesiones. Esto se obtiene a partir de una simple encuesta de costos médicos para el tratamiento de lesiones que incluyen estadía en un hospital, honorarios de médicos y medicamentos. El número promedio de lesiones totales por unidad de área de edificios derrumbados es $0.0168 / \mathrm{m}^{2}$ (se calcula dividiendo el número reportado de lesiones en el terremoto de 1985 en la Ciudad de México entre el área total estimada de edificios derrumbados, se considera tomando un promedio de la gravedad de lesiones leves y severas), (De León y Ang, 1994). Con el número de lesiones posibles, se realiza el cálculo del costo actual de gastos médicos mediante datos estadísticos y se multiplica por el número de lesionados.

$C_{f}=$ Costo por pérdida de vidas. El costo por pérdida de vidas se calculó con la vida productiva remanente, y el ingreso anual promedio para México y las muertes por m² $N_{D}$ (De León y Ang, 1994).

$$
N_{D}=45.48+5.53174 A^{2}
$$

donde A es el área de la planta del edificio.

Las estimaciones anteriores son condicionales a la ocurrencia del colapso del edificio. Por ello, el costo esperado de lesiones y fatalidades se obtienen multiplicando estas cantidades por la probabilidad de falla, que representa el colapso del edificio. En otros estudios, se presentan funciones de costo que dependen del nivel de daño y allí sí es pertinente el establecer coeficientes de variación o rangos de dispersión. En este caso, la incertidumbre asociada con estos valores condicionales depende de la aproximación en las cifras de la ecuación 14 y lo reducido de la muestra de 30 edificios colapsados en el temblor de 1985 (Ang y De León, 1994), empleados para estimar la tasa de lesiones entre el área de las plantas de los mismos. De acuerdo con los datos de esos edificios se estima un rango de variación de $\pm 40 \%$.

\section{CASOS DE ESTUDIO}

\section{Descripción de las escuelas}

Se analizaron 2 escuelas en el Estado de Morelos ya que son tipo U2C de 2 niveles, este tipo de escuelas son escuelas tipo que se encuentran en zonas sísmicas $\mathrm{B}, \mathrm{C}$ y $\mathrm{D}$, a base de marcos rígidos de concreto reforzado y muros de mampostería. Estas escuelas son de las pocas donde existen reportes fotográficos de los daños causados por el sismo del 2017. Además, dichas escuelas sufrieron una variedad de tipos de daños, desde daños en columnas, hasta daños moderados en trabes, losas y muros. Para la investigación, convenían estos tipos de daños para aplicar las diferentes propuestas de reparación. Si se hubieran tenido sólo daños menores, se ocuparía una reparación no estructural, y se requería edificios con daño en elementos estructurales y en muros.

\section{$\underline{\text { Yautepec }}$}

Los daños a la infraestructura educativa conforme a un comunicado oficial emitido por la Secretaría de Educación Pública (SEP), reportó 906 escuelas con daños menores que solo requerían reparaciones por daños menores como aplanados, caída de plafones, vidrios rotos. En la escuela aparentemente no se aprecian daños estructurales importantes, sin embargo, por alguna decisión gubernamental, la escuela fue demolida para ser construida nuevamente (García y otros, 2018), (figura 2). 


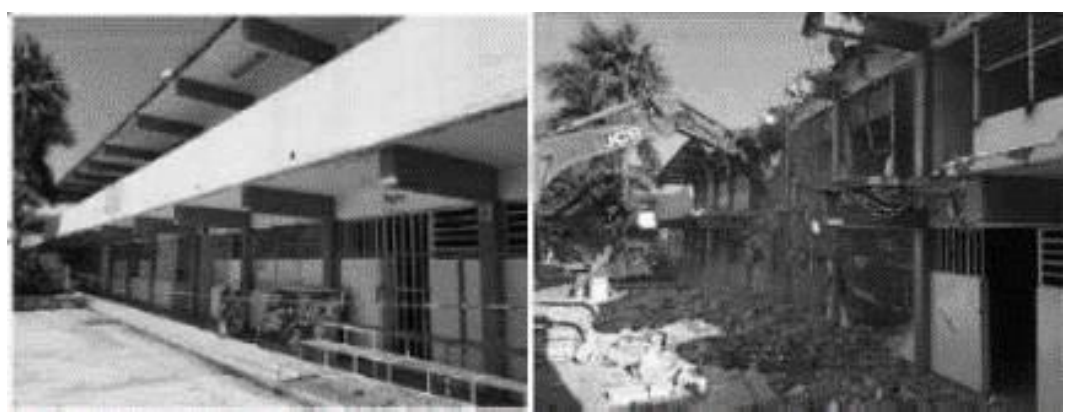

Figura 2. Escuela en Yautepec con daños

Yautepec se encuentra en el Estado de Morelos. La escuela consta de marcos de concreto reforzado de $\mathrm{f}^{\prime} \mathrm{c}=250 \mathrm{~kg} / \mathrm{cm}^{2}$ con muros de mampostería de $12 \mathrm{~cm}$ de espesor, con losa maciza de $12 \mathrm{~cm}$, y área de cada planta de $432.48 \mathrm{~m}^{2}$, con 12 crujías del lado largo de $3.19 \mathrm{~m}$, y una de $4 \mathrm{~m}$ (figura 3 ), en las figuras 4 , 5, 8, 9 se muestran con líneas punteadas los muros que son de mampostería de $12 \mathrm{~cm}$ de espesor.

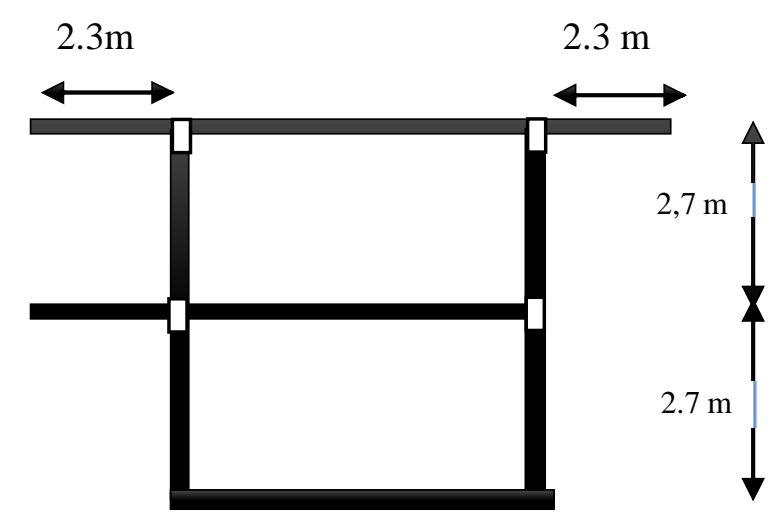

Figura 3. Vista de elevación, dirección transversal

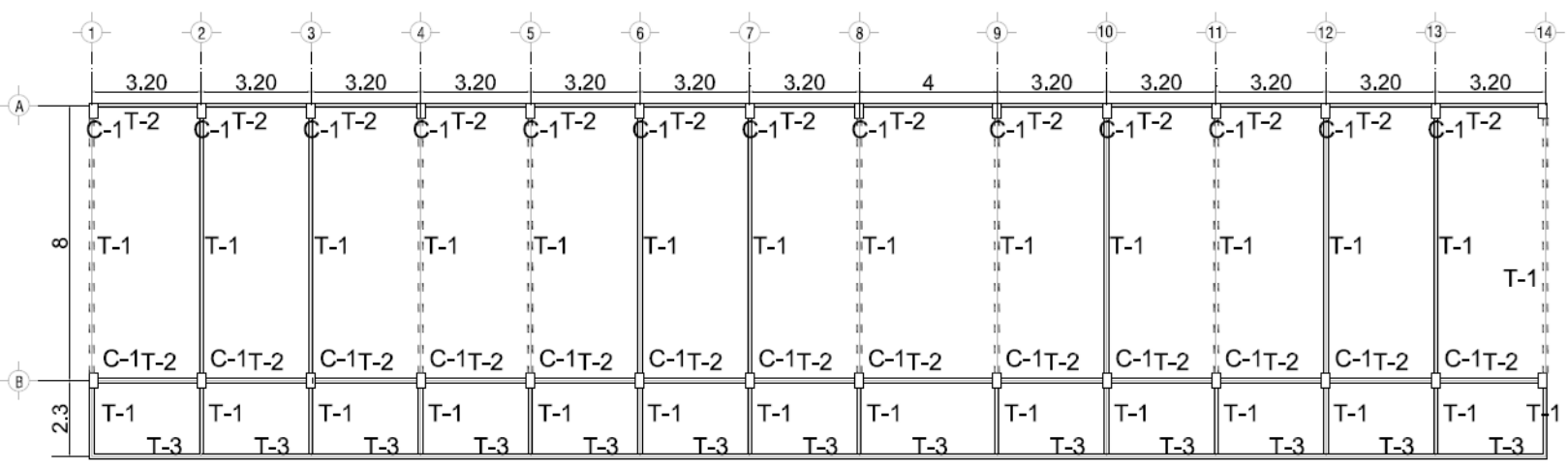

Figura 4. Vista de elementos estructurales. Planta baja 


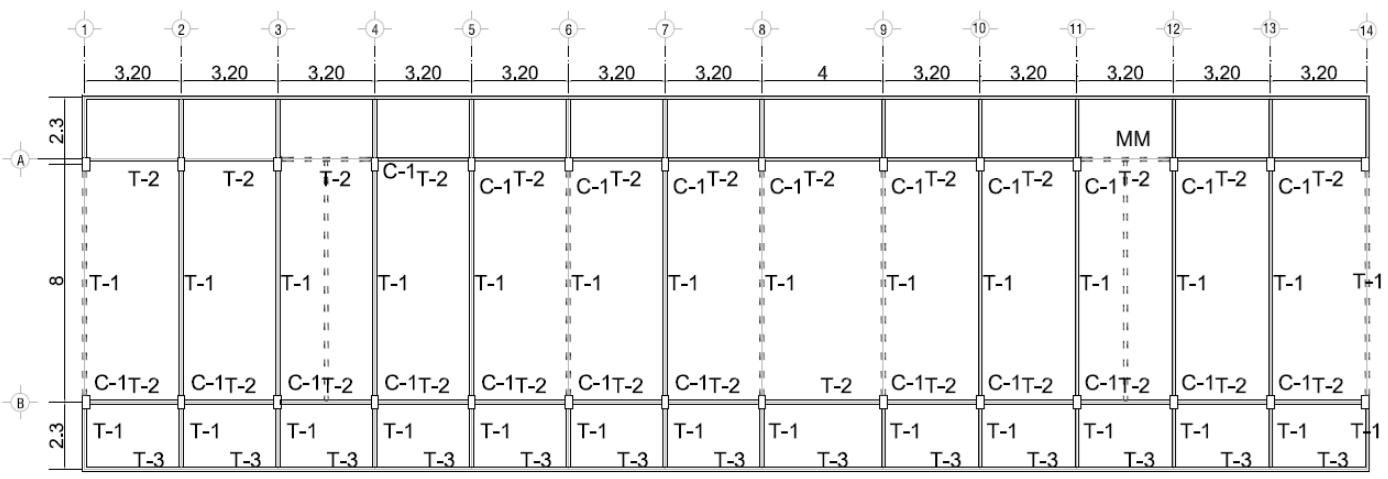

Figura 5. Vista de elementos estructurales. Planta alta

\section{$\underline{\text { Tlatenchi }}$}

En Tlatenchi, Estado de Morelos, a 15 minutos aproximadamente del centro de Jojutla, se encuentra la escuela primaria "Ignacio Zaragoza", la cual sufrió daños después del sismo del 19 de septiembre del 2017. Se trata de un prototipo U2C, se reportó con daños moderados, os daños presentados corresponden a fallas por tensión diagonal en muros y fallas por corte en columnas, la estructura fue demolida y ya estaba en construcción una nueva con el mismo prototipo, pero con normas vigentes (figura 6).
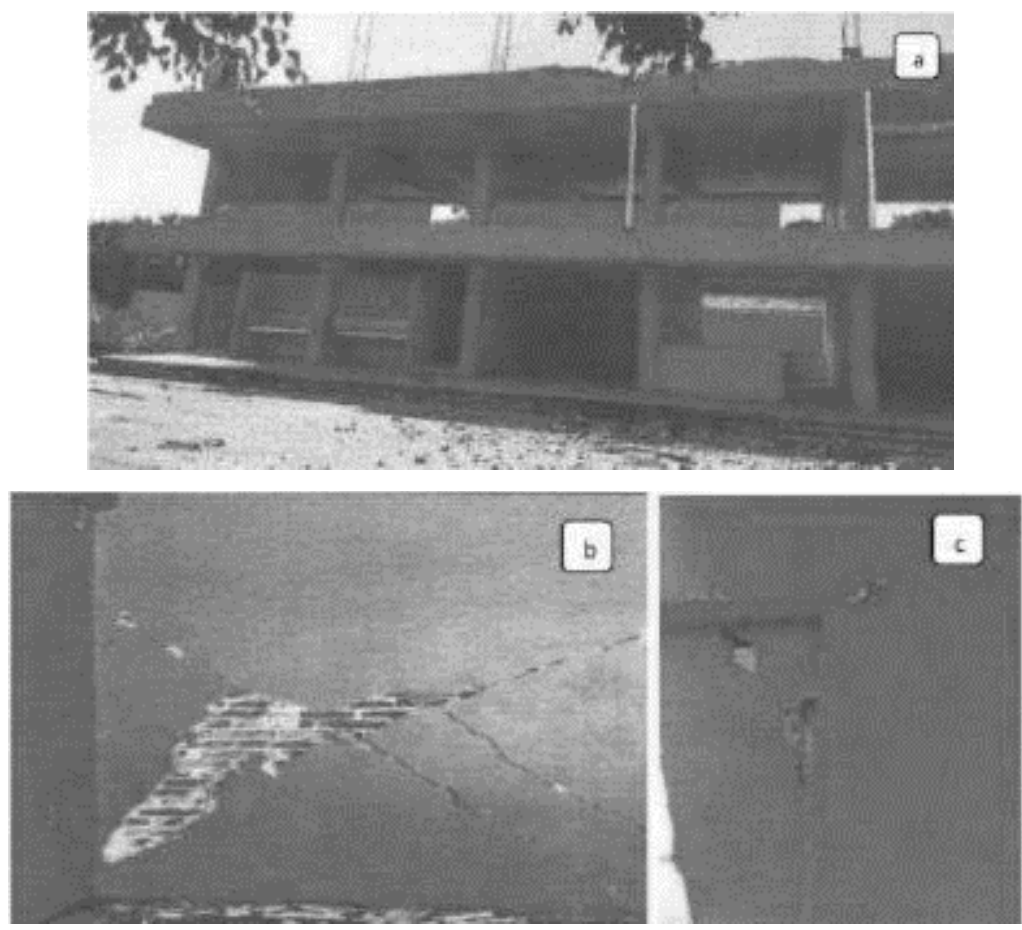

Figura 6. Escuela en Tlatenchi con daños

La escuela constaba de marcos de concreto reforzado de $\mathrm{f}^{\prime} \mathrm{c}=250 \mathrm{~kg} / \mathrm{cm}^{2}$ con muros de mampostería de $12 \mathrm{~cm}$ de espesor, con losa maciza de $12 \mathrm{~cm}$, y área de cada planta de $301.92 \mathrm{~m}^{2}$, con 8 crujías del lado largode $3.19 \mathrm{~m}$, y una de $4 \mathrm{~m}$ (figura 7). Asimismo, en las figuras 8 y 9 se muestran con líneas punteadas los muros que son de mampostería de $12 \mathrm{~cm}$ de espesor. 


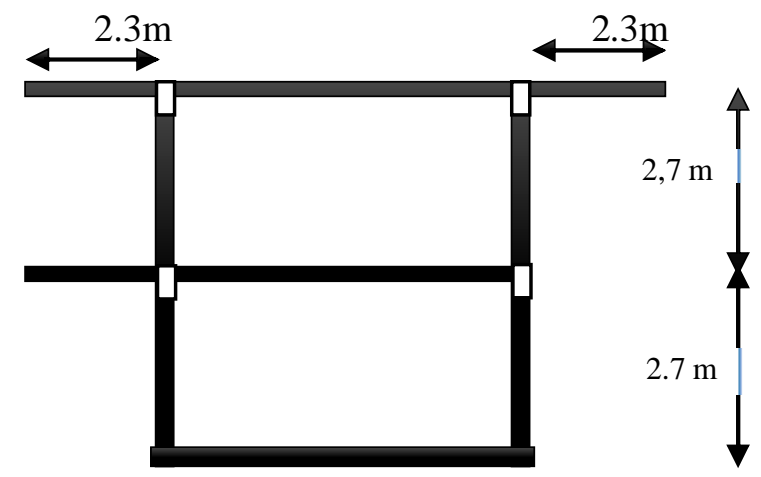

Figura 7. Vista de elevación, dirección transversal

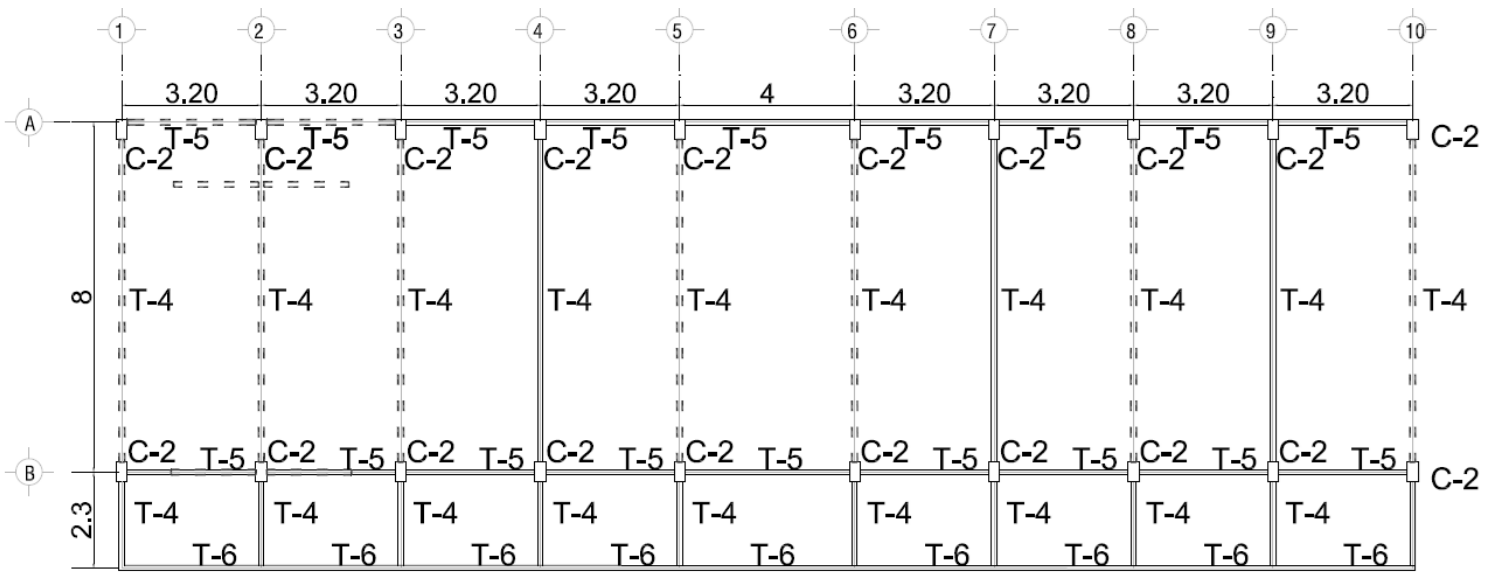

Figura 8. Vista de elementos estructurales. Planta baja

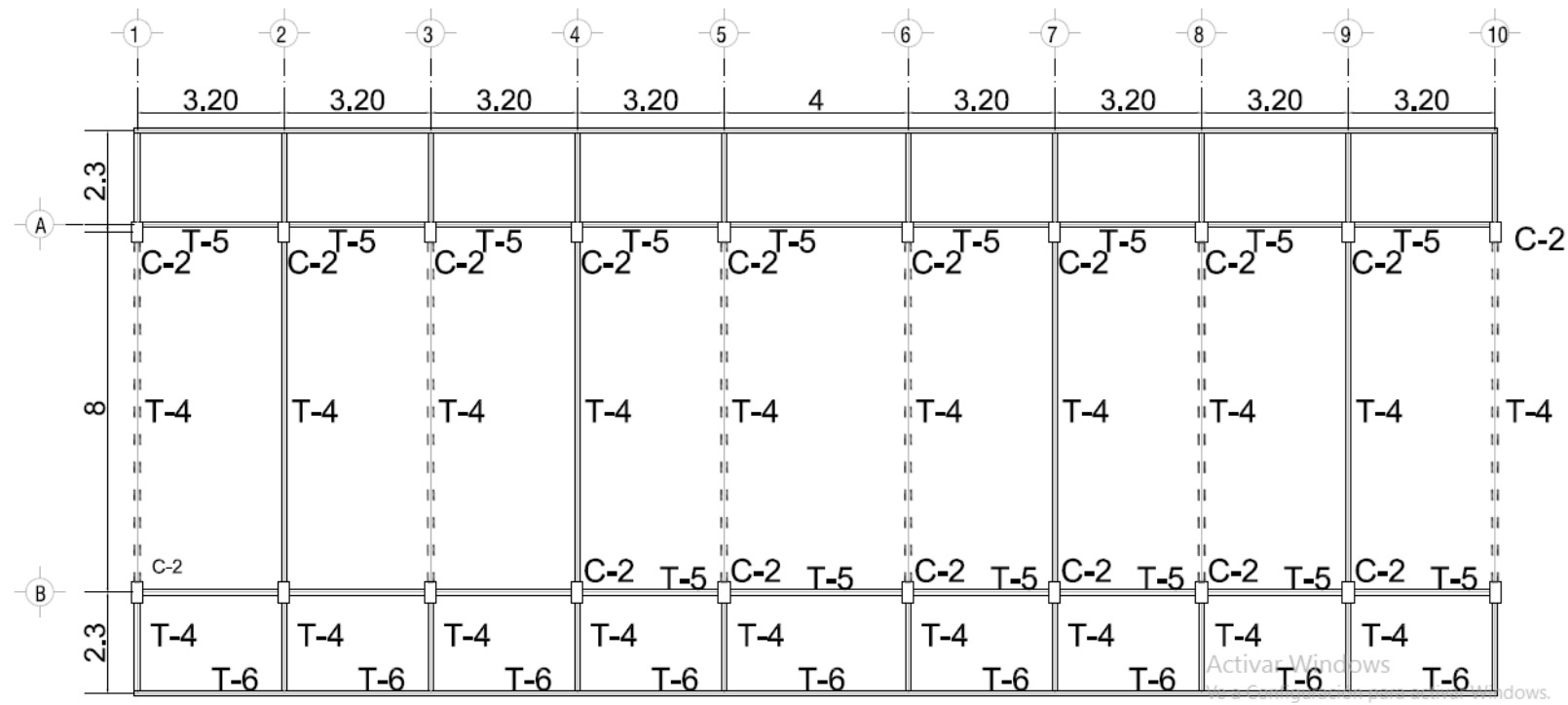

Figura 9. Vista de elementos estructurales. Planta alta

En la tabla 2 se muestran las dimensiones de las secciones de las columnas y de las trabes, así como el espesor de los muros de mampostería (MM). 
Tabla 2. Dimensiones de elementos estructurales

\begin{tabular}{|c|c|c|c|}
\hline $\begin{array}{l}\text { Elemento } \\
\text { estructural }\end{array}$ & $\begin{array}{l}\text { Dimensiones } \\
(\mathrm{cm})\end{array}$ & Armado Yautepec & Armado Tlatenchi \\
\hline $\mathrm{C}-1$ & $25 \times 45$ & 8 \#8, E\#3 @ 15 & 8 \#8, E\#3 @ 15 \\
\hline $\mathrm{C}-2$ & $25 \times 45$ & 8 \#8, E\#3 @ 15 & 8 \#8, E\#3 @ 15 \\
\hline $\mathrm{T}-1$ & $25 \times 55$ & $6 \# 5$, E\#3 @15 & 6 \#5, E\#3 @15 \\
\hline $\mathrm{T}-2$ & $20 \times 55$ & $6 \# 5$, E\#3 @15 & 6 \#5, E\#3 @15 \\
\hline $\mathrm{T}-3$ & $12 \times 55$ & $6 \# 5$, E\#3 @15 & 6\#5, E\#3 @15 \\
\hline $\mathrm{T}-4$ & $25 \times 55$ & 6\#5, E\#3 @15 & 6 \#5, E\#3 @15 \\
\hline $\mathrm{T}-5$ & $20 \times 55$ & $6 \# 5$, E\#3 @15 & 6 \#5, E\#3 @15 \\
\hline T-6 & $12 \times 55$ & $6 \# 5$, E\#3 @15 & 6\#5, E\#3 @15 \\
\hline MM & & $\mathrm{t}=12 \mathrm{~cm}$ & $\mathrm{t}=12 \mathrm{~cm}$ \\
\hline
\end{tabular}

Se procesaron los datos de las escuelas, como dimensiones en planta, secciones de los elementos, el análisis de carga viva y muerta, y posteriormente, se identificó el registro sísmico más cercano a la escuela para el sismo del 19 de septiembre del 2017. En este caso, para ambos, fue un registro sísmico en YAUTEPEC (figura 10), con una distancia epicentral a la estación de $63.16 \mathrm{~km}$. La distancia que existe entre la estación sísmica y la escuela de Yautepec es de $31.5 \mathrm{~km}$ y para Tlatenchi de $24.72 \mathrm{~km}$. La duración de la fase intensa es de aproximadamente $50 \mathrm{~s}$ y la aceleración pico es aproximadamente $0.2 \mathrm{~g}$. El espectro de respuesta como se ve en la figura 11. Para conocer cómo se comportó la estructura durante el sismo, después, se modeló en el Programa PERFORM- 3D. Los registros acelerográficos proporcionados han sido producto de las labores de instrumentación y procesamiento de la unidad de instrumentación Sísmica del Instituto de Ingeniería de la UNAM (II-UNAM, 2021).

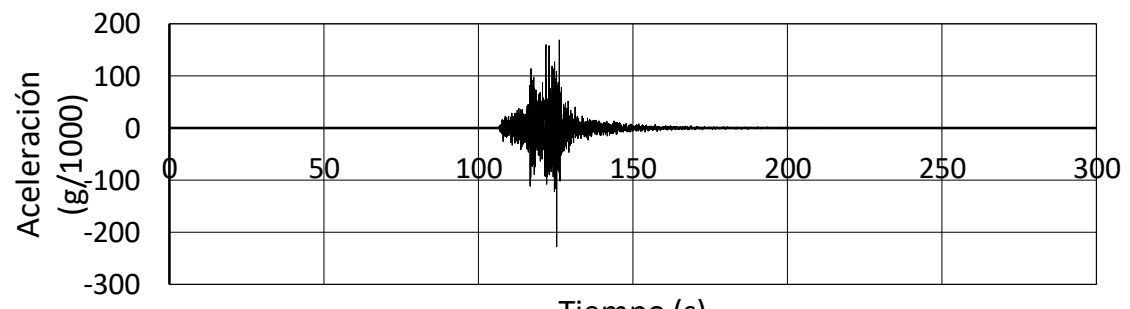

Tiempo (s)

Figura 10. Acelerograma estación YAUTEPEC (Canal -2, N00E). Dirección longitudinal

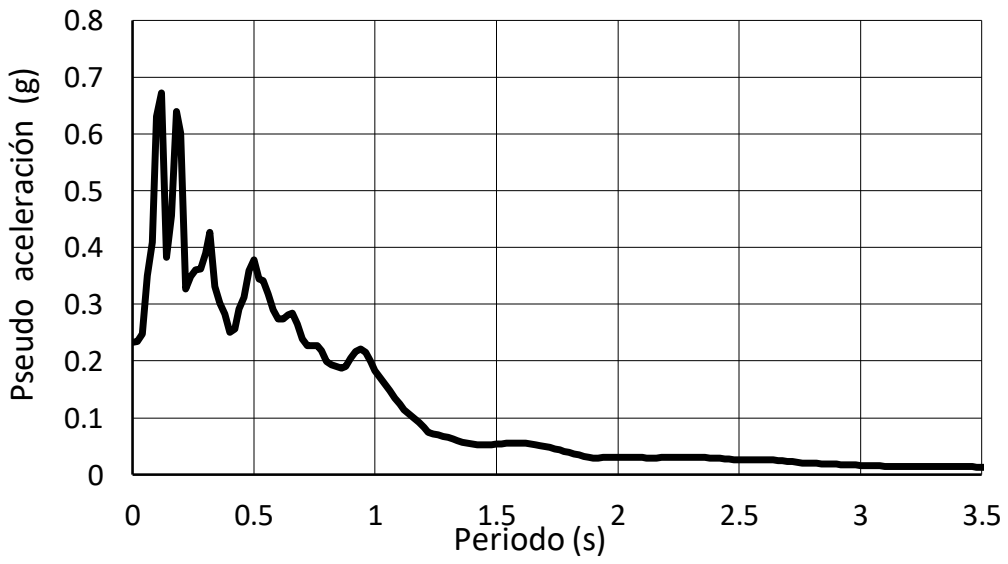

Figura 11. Espectro de respuesta, estación YAUTEPEC 
Posteriormente, se realiza el análisis no lineal de la estructura a partir de registro sísmico de Yautepec. En la figura 12 se muestra un ejemplo de los diagramas momento - curvatura que se tuvieron que obtener para el análisis no lineal. Se usó el modelo histerético trilineal ya que presenta una mejor aproximación de las características de rigidez sometidos a flexión, en cambio con el modelo bilineal no considera la degradación de la rigidez en la etapa de descarga (figura 13). Para las columnas se analizó la fuerza combinada bajo flexión biaxial, con carga axial, para secciones rectangulares, hay soluciones basadas en capacidades de la sección sobre los ejes principales (Furlong y Hsu, 2004), y que está publicado en (ASCE 41-13, 2019).
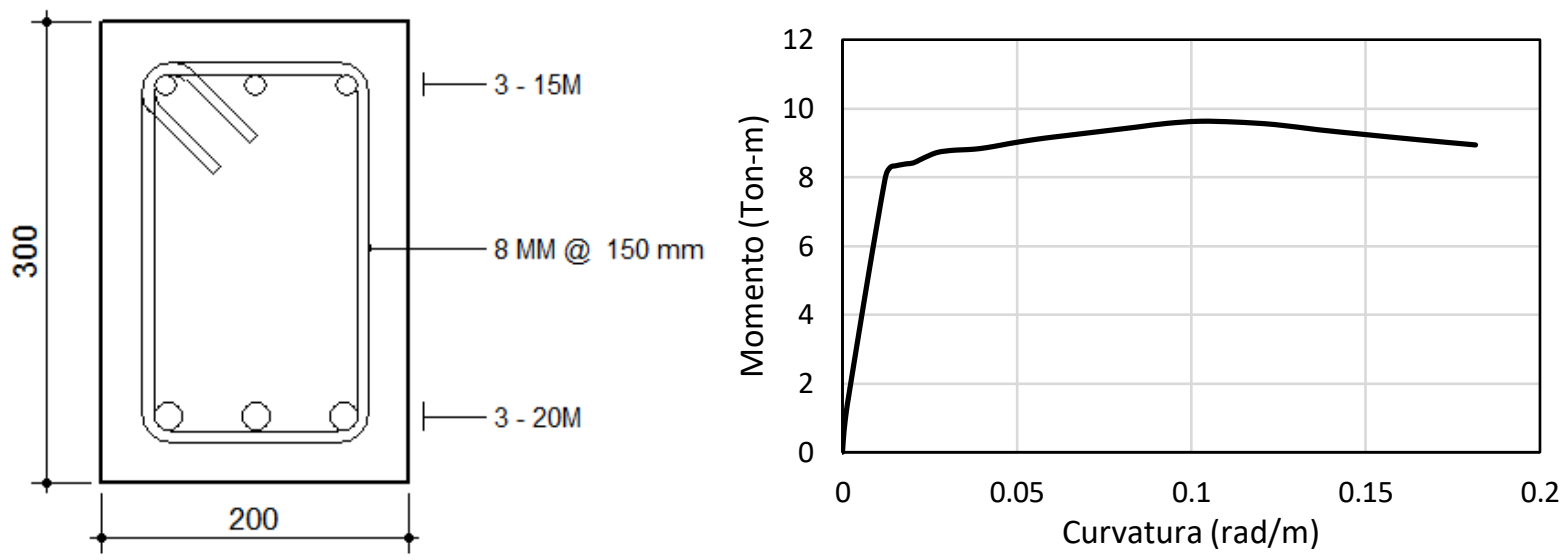

Figura 12. Sección transversal y diagrama momento curvatura

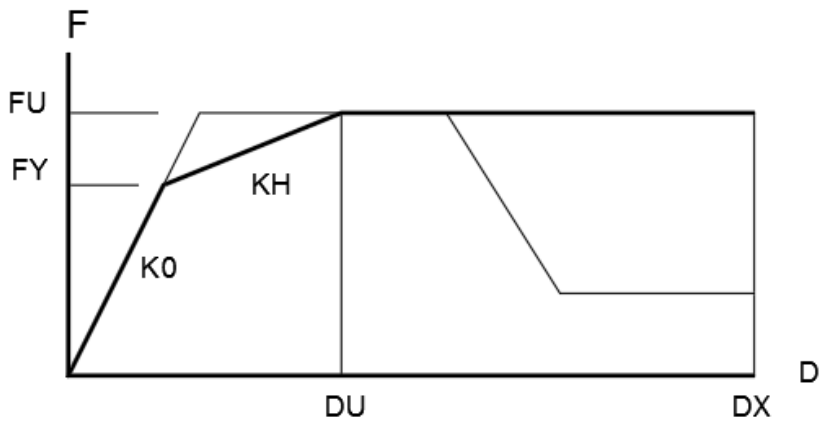

Figura 13. Modelo trilineal

Posteriormente, se obtienen las probabilidades de falla de las columnas y trabes. En las tablas 3, 4, 5 y 6 , se muestra una parte de los índices de confiabilidad de las columnas y trabes de ambas escuelas.

Una vez identificados los refuerzos, se procedió a realizar el análisis de la respuesta de las escuelas, con el espectro de respuesta, para obtener los elementos mecánicos y, de ahí, las probabilidades de falla e índices de confiabilidad. Las tablas 3 y 4 muestran los resultados para columnas de Yautepec y Tlatenchi, respectivamente. Las tablas 5 y 6 para las trabes de Yautepec y Tlatenchi, respectivamente. En el caso de las columnas, el índice $\beta$ se obtuvo, como se describió antes, de simulaciones de Monte Carlo con el estado límite que incluye la interacción entre flexión y compresión y simulando variables lognormales. Para trabes, se usaron las ecuaciones 1 a 4 . En todos los casos, para la probabilidad de falla de la estructura, se consideraron las probabilidades de falla locales, o de los miembros, de acuerdo con las ecuaciones 5 a 9. 
Tabla 3. Probabilidad de falla de columnas. Escuela Yautepec

\begin{tabular}{cccccccc}
\hline $\begin{array}{c}\text { Columna } \\
\text { Elemento \# }\end{array}$ & P (ton) & $\begin{array}{c}\text { Actuante } \\
\text { M3 }\end{array}$ & $\begin{array}{c}\text { M2 } \\
\text { (ton-m) }\end{array}$ & $\begin{array}{c}\text { R (ton-m) } \\
\text { (ton) }\end{array}$ & $\begin{array}{c}\text { M3 } \\
\text { (ton-m) }\end{array}$ & $\begin{array}{c}\text { M2 } \\
\text { (ton-m) }\end{array}$ & $\beta$ \\
\hline 1 & 18.22 & 5.17 & 0.07 & 229.25 & 23.83 & 10.89 & 4.95 \\
2 & 6.31 & 5.33 & 0.10 & 229.25 & 23.83 & 10.89 & 4.86 \\
11 & 18.24 & 5.15 & 0.08 & 229.25 & 23.83 & 10.89 & 4.96 \\
12 & 6.30 & 5.33 & 0.11 & 229.25 & 23.83 & 10.89 & 4.86 \\
17 & 21.71 & 5.18 & 0.06 & 229.25 & 23.83 & 10.89 & 4.95 \\
18 & 10.03 & 5.47 & 0.08 & 229.25 & 23.83 & 10.89 & 4.77 \\
19 & 17.08 & 5.20 & 0.09 & 229.25 & 23.83 & 10.89 & 4.93 \\
20 & 10.04 & 5.48 & 0.09 & 229.25 & 23.83 & 10.89 & 4.77 \\
25 & 21.55 & 5.19 & 0.07 & 229.25 & 23.83 & 10.89 & 4.94 \\
26 & 9.97 & 5.59 & 0.108 & 229.25 & 23.82 & 10.88 & 4.70 \\
\hline
\end{tabular}

Tabla 4. Probabilidad de falla de columnas. Escuela Tlatenchi

\begin{tabular}{cccccccc}
\hline $\begin{array}{c}\text { Columna } \\
\text { Elemento \# }\end{array}$ & P (ton) & $\begin{array}{c}\text { Actuante } \\
\text { (ton-m) }\end{array}$ & $\begin{array}{c}\text { M2 } \\
\text { (ton-m) }\end{array}$ & P (ton) & $\begin{array}{c}\text { M3 } \\
\text { (ton-m) }\end{array}$ & $\begin{array}{c}\text { M2 } \\
\text { (ton-m) }\end{array}$ & $\beta$ \\
\hline 3 & 5.13 & 10.91 & 0.78 & 229.25 & 23.83 & 10.89 & 2.59 \\
4 & 1.67 & 8.86 & 0.24 & 229.25 & 23.83 & 10.89 & 3.25 \\
5 & 5.12 & 10.91 & 0.50 & 229.25 & 23.83 & 10.89 & 2.59 \\
6 & 1.67 & 8.83 & 0.75 & 229.25 & 23.83 & 10.89 & 3.26 \\
25 & 20.27 & 10.91 & 0.88 & 229.25 & 23.83 & 10.89 & 2.59 \\
26 & 9.22 & 8.61 & 0.32 & 229.25 & 23.83 & 10.89 & 3.34 \\
27 & 16.06 & 10.91 & 0.66 & 229.25 & 23.83 & 10.89 & 2.59 \\
28 & 9.39 & 8.57 & 0.90 & 229.25 & 23.83 & 10.89 & 3.36 \\
33 & 4.92 & 10.51 & 0.87 & 229.25 & 23.83 & 10.89 & 2.71 \\
34 & 1.637 & 8.436 & 0.31 & 229.25 & 23.827 & 10.887 & 3.40 \\
\hline
\end{tabular}

Tabla 5. Probabilidad de falla de trabes. Escuela Yautepec

\begin{tabular}{|c|c|c|c|c|c|c|c|c|c|}
\hline \multirow{2}{*}{$\begin{array}{c}\text { Trabe } \\
\text { Elemento \# }\end{array}$} & \multicolumn{2}{|c|}{ Actuante } & \multicolumn{2}{|c|}{ Resistente } & \multirow{2}{*}{$\begin{array}{l}\text { Mact / } \\
\text { M res }\end{array}$} & \multirow{2}{*}{$\begin{array}{l}\text { Vact / } \\
\text { V res }\end{array}$} & \multirow{2}{*}{$\begin{array}{c}\text { Mediana } \\
\text { Ma }\end{array}$} & \multirow{2}{*}{$\begin{array}{c}\text { Mediana } \\
\mathrm{Mr}\end{array}$} & \multirow[b]{2}{*}{$\beta$} \\
\hline & $\begin{array}{c}\mathrm{M} \\
\text { (ton-m) }\end{array}$ & $\begin{array}{c}\mathrm{V} \\
\text { (ton) }\end{array}$ & $\begin{array}{c}\mathrm{M} \\
\text { (ton-m) }\end{array}$ & $\begin{array}{c}\mathrm{V} \\
\text { (ton) }\end{array}$ & & & & & \\
\hline 23 & 8.64 & 5.65 & 18.75 & 18.99 & 0.46 & 0.3 & 7.93 & 18.66 & 2.71 \\
\hline 24 & 6.42 & 5.17 & 18.75 & 18.99 & 0.34 & 0.27 & 5.89 & 18.66 & 3.65 \\
\hline 31 & 8.69 & 5.65 & 18.75 & 18.99 & 0.46 & 0.3 & 7.97 & 18.66 & 2.69 \\
\hline 32 & 6.43 & 5.18 & 18.75 & 18.99 & 0.34 & 0.27 & 5.9 & 18.66 & 3.64 \\
\hline 39 & 8.69 & 5.65 & 18.75 & 18.99 & 0.46 & 0.3 & 7.97 & 18.66 & 2.69 \\
\hline 40 & 6.44 & 5.18 & 18.75 & 18.99 & 0.34 & 0.27 & 5.9 & 18.66 & 3.64 \\
\hline 47 & 8.77 & 5.63 & 18.75 & 18.99 & 0.47 & 0.3 & 8.05 & 18.66 & 2.66 \\
\hline 48 & 6.51 & 5.18 & 18.75 & 18.99 & 0.35 & 0.27 & 5.98 & 18.66 & 3.6 \\
\hline 55 & 8.66 & 2.34 & 18.75 & 18.99 & 0.46 & 0.12 & 7.94 & 18.66 & 2.7 \\
\hline 56 & 5.58 & 2.59 & 18.75 & 18.99 & 0.3 & 0.14 & 5.12 & 18.66 & 4.09 \\
\hline 63 & 8.71 & 2.34 & 18.75 & 18.99 & 0.46 & 0.12 & 7.99 & 18.66 & 2.68 \\
\hline
\end{tabular}


Tabla 6. Probabilidad de falla de trabes. Escuela Tlatenchi

\begin{tabular}{|c|c|c|c|c|c|c|c|c|c|}
\hline \multirow{2}{*}{$\begin{array}{c}\text { Trabe } \\
\text { Elemento \# }\end{array}$} & \multicolumn{2}{|c|}{ Actuante } & \multicolumn{2}{|c|}{ Resistente } & \multirow{2}{*}{$\begin{array}{l}\text { Mact/ } \\
\text { M res }\end{array}$} & \multirow{2}{*}{$\begin{array}{c}\text { Vact/ V } \\
\text { res }\end{array}$} & \multirow{2}{*}{$\begin{array}{c}\text { Mediana } \\
\text { Ma }\end{array}$} & \multirow{2}{*}{$\begin{array}{c}\text { Mediana } \\
\text { Mr }\end{array}$} & \multirow{2}{*}{$\beta$} \\
\hline & $\begin{array}{c}\mathrm{M} \\
\text { (ton-m) }\end{array}$ & $\begin{array}{c}\mathrm{V} \\
\text { (ton) }\end{array}$ & $\begin{array}{c}\mathrm{M} \\
\text { (ton-m) }\end{array}$ & $\begin{array}{c}\mathrm{V} \\
\text { (ton) }\end{array}$ & & & & & \\
\hline 9 & 10.464 & 2.683 & 18.75 & 18.99 & 0.01 & 0.51 & 9.6 & 18.66 & 2.1 \\
\hline 10 & 8.794 & 2.579 & 18.75 & 18.99 & 0.01 & 0.51 & 8.07 & 18.66 & 2.65 \\
\hline 31 & 10.463 & 5.672 & 18.75 & 18.99 & 0.02 & 0.51 & 9.6 & 18.66 & 2.1 \\
\hline 32 & 8.56 & 5.192 & 18.75 & 18.99 & 0.01 & 0.51 & 7.85 & 18.66 & 2.74 \\
\hline 39 & 10.462 & 5.65 & 18.75 & 18.99 & 0.02 & 0.51 & 9.6 & 18.66 & 2.1 \\
\hline 40 & 8.454 & 5.188 & 18.75 & 18.99 & 0.01 & 0.51 & 7.76 & 18.66 & 2.78 \\
\hline 47 & 10.461 & 5.662 & 18.75 & 18.99 & 0.02 & 0.51 & 9.6 & 18.66 & 2.1 \\
\hline 48 & 8.26 & 5.197 & 18.75 & 18.99 & 0.01 & 0.51 & 7.58 & 18.66 & 2.85 \\
\hline 55 & 10.46 & 2.661 & 18.75 & 18.99 & 0.01 & 0.51 & 9.6 & 18.66 & 2.1 \\
\hline 56 & 8.159 & 2.602 & 18.75 & 18.99 & 0.01 & 0.51 & 7.49 & 18.66 & 2.89 \\
\hline 63 & 10.458 & 2.653 & 18.75 & 18.99 & 0.01 & 0.51 & 9.59 & 18.66 & 2.1 \\
\hline
\end{tabular}

Las tablas anteriores muestran qué elementos fueron los más dañados, en este caso son los que tengan un índice de confiabilidad menor. Después de haber identificado los elementos se procede a las opciones de reparación de las escuelas.

\section{ALTERNATIVAS DE REPARACIÓN}

Se propone una intervención de refuerzo en los elementos más críticos o con probabilidad de falla mayor, se propusieron 3 alternativas de reparación, considerando que en un sismo futuro de igual o mayor magnitud al sismo que lo halla dañado originalmente, se mantenga con un índice de confiabilidad mayor a 3 , las propuestas son encamisado con concreto reforzado, encamisado con ángulos y soleras de acero y colocación de muros de concreto. Estas alternativas deben disminuir las probabilidades de falla de los elementos.

\section{Encamisado de concreto}

Se comienza con las columnas con mayor probabilidad de falla de ambas escuelas (figura 14). A continuación, se muestra el ejemplo de una columna que tuvo daño. Se usó como apoyo la guía técnica para la rehabilitación sísmica de edificios escolares de la Ciudad de México (INIFED, 2021)

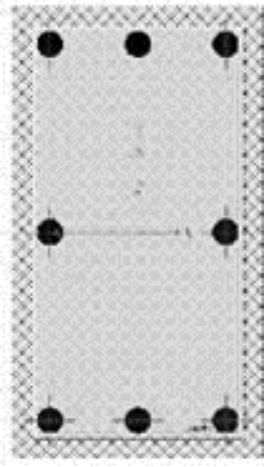

Figura 14. Columna dañada

Posteriormente, se procedió a la alternativa de refuerzo que es mediante el encamisado de columnas (figura15), en este caso se modeló en Sap 2000, en "Section Designer". Como se ve a continuación, a la 
sección se le aumentó 10 varillas \#5 aparte de las que tenía originalmente, y f'c de $\left(200 \mathrm{~kg} / \mathrm{cm}^{2}\right)$, ya que, con este armado, el índice de confiabilidad ya supera al permisible de acuerdo con el Eurocódigo.
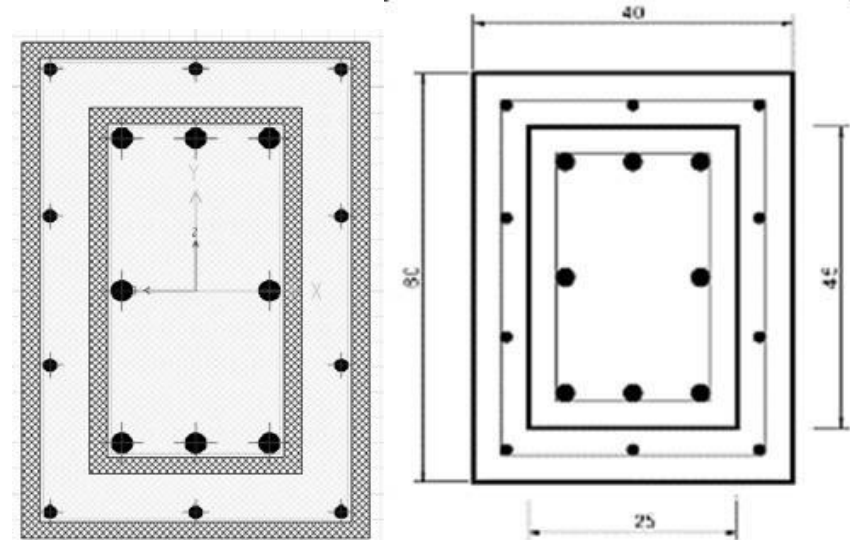

Figura 15. Columna analizada en SAP con concreto reforzado (cotas en $\mathrm{cm}$ )

Se obtuvieron los diagramas de interacción, para los 2 sentidos, M3 y M2, como se muestra en las figuras 16 y 17.

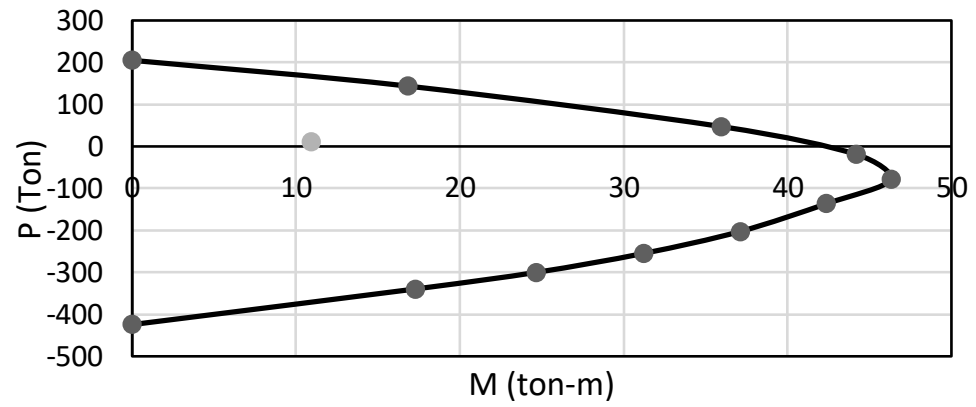

Figura 16. Diagrama interacción M3

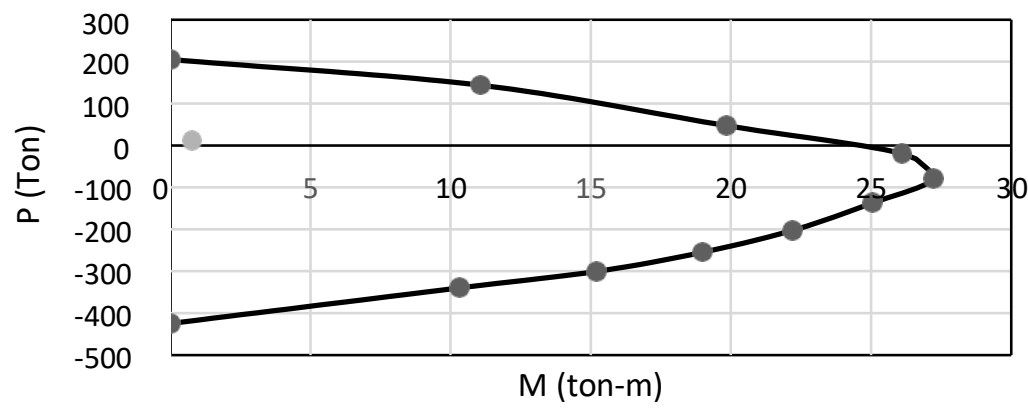

Figura 17. Diagrama interacción M2

En las tablas 7 y 8 se muestran los resultados de la alternativa para columnas de concreto reforzado. Nuevamente, se empleó el espectro de respuesta para cada sitio, con las nuevas dimensiones en los elementos reparados. 
Tabla 7. Probabilidad de falla de columnas (encamisado concreto). Escuela Yautepec

\begin{tabular}{cccccccc}
$\begin{array}{c}\text { Columna } \\
\text { Elemento \# }\end{array}$ & \multicolumn{3}{c}{ Actuante } & \multicolumn{3}{c}{ Resistente } \\
& P (ton) & $\begin{array}{c}\text { M3 } \\
\text { (ton-m) }\end{array}$ & $\begin{array}{c}\text { M2 } \\
\text { (ton-m) }\end{array}$ & P (ton) & $\begin{array}{c}\text { M3 } \\
\text { (ton-m) }\end{array}$ & $\begin{array}{c}\text { M2 } \\
\text { (ton-m) }\end{array}$ \\
\hline 1 & 18.22 & 5.17 & 0.07 & 229.25 & 23.83 & 10.89 & 4.95 \\
2 & 6.31 & 5.33 & 0.10 & 229.25 & 23.83 & 10.89 & 4.86 \\
11 & 18.24 & 5.15 & 0.08 & 229.25 & 23.83 & 10.89 & 4.96 \\
12 & 6.30 & 5.33 & 0.11 & 229.25 & 23.83 & 10.89 & 4.86 \\
17 & 21.71 & 5.18 & 0.06 & 229.25 & 23.83 & 10.89 & 4.95 \\
18 & 10.03 & 5.47 & 0.08 & 229.25 & 23.83 & 10.89 & 4.77 \\
19 & 17.08 & 5.20 & 0.09 & 229.25 & 23.83 & 10.89 & 4.93 \\
20 & 10.04 & 5.48 & 0.09 & 229.25 & 23.83 & 10.89 & 4.77 \\
25 & 21.55 & 5.19 & 0.07 & 229.25 & 23.83 & 10.89 & 4.94 \\
26 & 9.97 & 5.59 & 0.11 & 229.25 & 23.83 & 10.89 & 4.71 \\
\hline
\end{tabular}

Tabla 8. Probabilidad de falla de columnas (encamisado concreto). Escuela Tlatenchi

\begin{tabular}{cccccccc}
$\begin{array}{c}\text { Columna } \\
\text { Elemento \# }\end{array}$ & \multicolumn{7}{c}{$\begin{array}{c}\text { Resistente } \\
\text { Actuante }\end{array}$} \\
& $\mathrm{P}$ (ton) & $\begin{array}{c}\text { M3 } \\
\text { (ton-m) }\end{array}$ & $\begin{array}{c}\text { M2 } \\
\text { (ton-m) }\end{array}$ & P (ton) & $\begin{array}{c}\text { M3 } \\
\text { (ton-m) }\end{array}$ & $\begin{array}{c}\text { M2 } \\
\text { (ton-m) }\end{array}$ \\
\hline 3 & 5.13 & 10.91 & 0.78 & 424.45 & 41.83 & 26.15 & 4.37 \\
4 & 1.67 & 8.86 & 0.24 & 229.25 & 23.83 & 10.89 & 3.85 \\
5 & 5.12 & 10.91 & 0.50 & 424.45 & 41.83 & 26.15 & 4.37 \\
6 & 1.67 & 8.83 & 0.75 & 229.25 & 23.83 & 10.89 & 3.86 \\
25 & 20.27 & 10.91 & 0.88 & 424.45 & 41.83 & 26.15 & 4.37 \\
26 & 9.22 & 8.61 & 0.32 & 229.25 & 23.83 & 10.89 & 3.94 \\
27 & 16.06 & 10.91 & 0.66 & 424.45 & 41.83 & 26.15 & 4.37 \\
28 & 9.39 & 8.57 & 0.90 & 229.25 & 23.83 & 10.89 & 3.96 \\
33 & 4.92 & 10.51 & 0.87 & 424.45 & 41.83 & 26.15 & 4.49 \\
34 & 1.64 & 8.44 & 0.31 & 229.25 & 23.83 & 10.89 & 4.00 \\
\hline
\end{tabular}

Se continua ahora, con el encamisado de las trabes más dañadas como se muestra en la figura 18. La guía técnica para la rehabilitación sísmica de edificios escolares de la ciudad de México (INIFED, 2021) menciona los recubrimientos mínimos, y armados del acero para la sección compuesta.

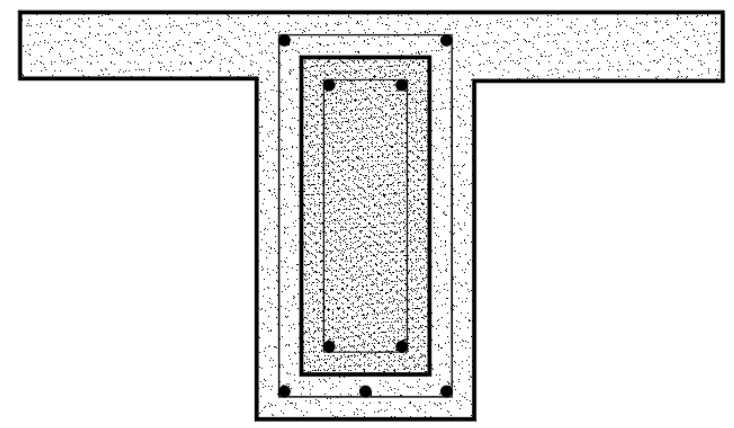

Figura 18. Encamisado de trabe de concreto reforzado (Instituto de Ingeniería UNAM, 2019) 
Después de consultar la guía técnica, se muestra a continuación el ejemplo de una trabe dañada. Se llegó a las dimensiones y refuerzo del acero mostradas en la figura 19 de forma que cumpla con el índice de confiabilidad permisible.
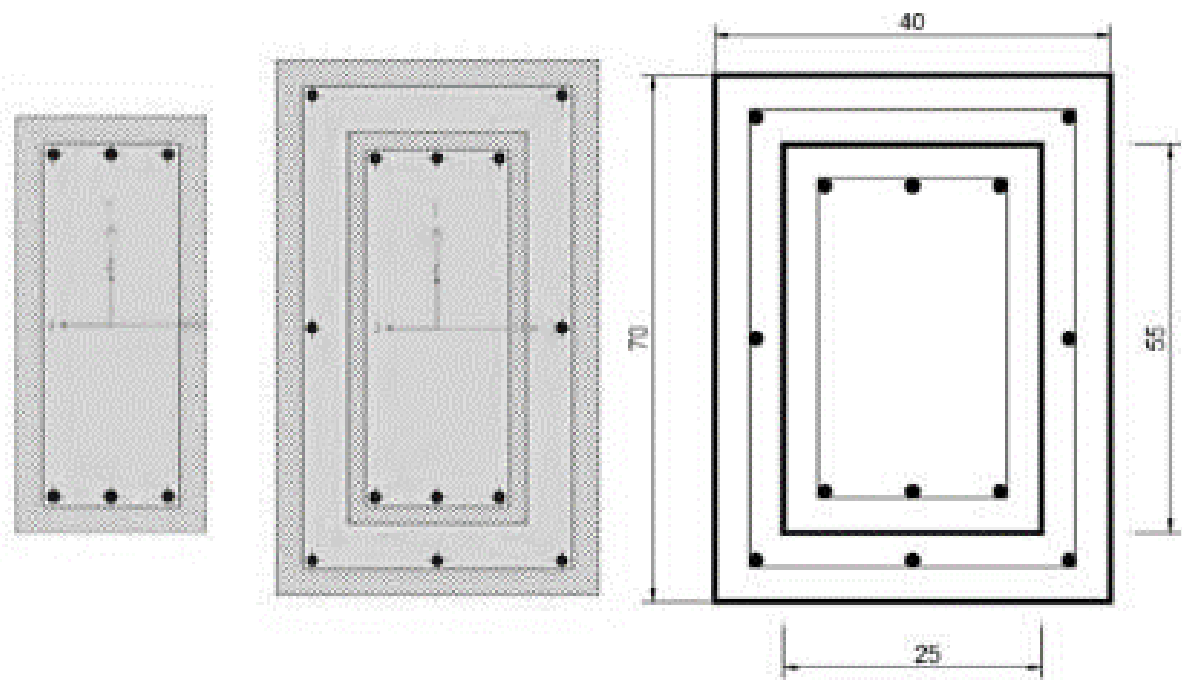

Figura 19. Encamisado de trabe (concreto reforzado, cotas $\mathrm{cm}$ )

En las tablas 9 y 10, se muestran los resultados de la alternativa 1 para trabes de concreto reforzado. Como se ha descrito, el índice de confiabilidad para trabes se obtiene de las ecuaciones 1 a 3.

Tabla 9. Índices de confiabilidad de trabes (encamisado de concreto). Escuela Yautepec

\begin{tabular}{|c|c|c|c|c|c|c|c|c|c|}
\hline \multirow{2}{*}{$\begin{array}{c}\text { Trabe } \\
\text { Elemento \# }\end{array}$} & \multicolumn{2}{|c|}{ Actuante } & \multicolumn{2}{|c|}{ Resistente } & \multirow{2}{*}{$\begin{array}{l}\text { Mact / } \\
\text { M res }\end{array}$} & \multirow{2}{*}{$\begin{array}{l}\text { Vact / } \\
\text { V res }\end{array}$} & \multirow{2}{*}{$\begin{array}{c}\text { Mediana } \\
\text { Ma }\end{array}$} & \multirow{2}{*}{$\begin{array}{c}\text { Mediana } \\
\text { Mr }\end{array}$} & \multirow{2}{*}{$\beta$} \\
\hline & $\begin{array}{c}\mathrm{M} \\
\text { (ton-m) }\end{array}$ & $\begin{array}{c}\mathrm{V} \\
\text { (ton) }\end{array}$ & $\begin{array}{c}\mathrm{M} \\
\text { (ton-m) }\end{array}$ & $\begin{array}{c}\mathrm{V} \\
\text { (ton) }\end{array}$ & & & & & \\
\hline 23 & 8.64 & 5.65 & 27.08 & 22.4 & 0.32 & 0.25 & 7.93 & 26.95 & 3.87 \\
\hline 24 & 6.42 & 5.17 & 18.75 & 18.99 & 0.34 & 0.27 & 5.89 & 18.66 & 3.85 \\
\hline 31 & 8.69 & 5.65 & 27.08 & 22.4 & 0.32 & 0.25 & 7.97 & 26.95 & 3.85 \\
\hline 32 & 6.43 & 5.18 & 18.75 & 18.99 & 0.34 & 0.27 & 5.9 & 18.66 & 3.84 \\
\hline 39 & 8.69 & 5.65 & 27.08 & 22.4 & 0.32 & 0.25 & 7.97 & 26.95 & 3.85 \\
\hline 40 & 6.44 & 5.18 & 18.75 & 18.99 & 0.34 & 0.27 & 5.9 & 18.66 & 3.84 \\
\hline 47 & 8.77 & 5.63 & 27.08 & 22.4 & 0.32 & 0.25 & 8.05 & 26.95 & 3.82 \\
\hline 48 & 6.51 & 5.18 & 18.75 & 18.99 & 0.35 & 0.27 & 5.98 & 18.66 & 3.8 \\
\hline 55 & 8.66 & 2.34 & 27.08 & 22.4 & 0.32 & 0.1 & 7.94 & 26.95 & 3.86 \\
\hline 56 & 5.58 & 2.59 & 18.75 & 18.99 & 0.3 & 0.14 & 5.12 & 18.66 & 4.09 \\
\hline 63 & 8.71 & 2.34 & 27.08 & 22.4 & 0.32 & 0.1 & 7.99 & 26.95 & 3.84 \\
\hline
\end{tabular}


Tabla 10. Índices de confiabilidad de trabes (encamisado de concreto). Escuela Tlatenchi

\begin{tabular}{|c|c|c|c|c|c|c|c|c|c|}
\hline \multirow{2}{*}{$\begin{array}{c}\text { Trabe } \\
\text { Elemento \# }\end{array}$} & \multicolumn{2}{|c|}{ Actuante } & \multicolumn{2}{|c|}{ Resistente } & \multirow{2}{*}{$\begin{array}{l}\text { Mact/ } \\
\text { M res }\end{array}$} & \multirow{2}{*}{$\begin{array}{l}\text { Vact/ } \\
\text { V res }\end{array}$} & \multirow{2}{*}{$\begin{array}{c}\text { Mediana } \\
\text { Ma }\end{array}$} & \multirow{2}{*}{$\begin{array}{c}\text { Mediana } \\
\text { Mr }\end{array}$} & \multirow{2}{*}{$\beta$} \\
\hline & $\begin{array}{c}\mathrm{M} \\
\text { (ton-m) }\end{array}$ & $\mathrm{V}$ (ton) & $\begin{array}{c}\mathrm{M} \\
\text { (ton-m) }\end{array}$ & $\mathrm{V}$ (ton) & & & & & \\
\hline 9 & 10.464 & 2.683 & 30.21 & 26.56 & 0.35 & 0.1 & 9.6 & 30.06 & 3.81 \\
\hline 10 & 8.794 & 2.579 & 30.21 & 26.56 & 0.29 & 0.1 & 8.07 & 30.06 & 4.16 \\
\hline 31 & 10.463 & 5.672 & 30.21 & 26.56 & 0.35 & 0.21 & 9.6 & 30.06 & 3.81 \\
\hline 32 & 8.56 & 5.192 & 30.21 & 26.56 & 0.28 & 0.2 & 7.85 & 30.06 & 4.24 \\
\hline 39 & 10.462 & 5.65 & 30.21 & 26.56 & 0.35 & 0.21 & 9.6 & 30.06 & 3.81 \\
\hline 40 & 8.454 & 5.188 & 30.21 & 26.56 & 0.28 & 0.2 & 7.76 & 30.06 & 4.28 \\
\hline 47 & 10.461 & 5.662 & 30.21 & 26.56 & 0.35 & 0.21 & 9.6 & 30.06 & 3.81 \\
\hline 48 & 8.26 & 5.197 & 30.21 & 26.56 & 0.27 & 0.2 & 7.58 & 30.06 & 4.36 \\
\hline 55 & 10.46 & 2.661 & 30.21 & 26.56 & 0.35 & 0.1 & 9.6 & 30.06 & 3.81 \\
\hline 63 & 10.458 & 2.653 & 30.21 & 26.56 & 0.35 & 0.1 & 9.59 & 30.06 & 3.81 \\
\hline
\end{tabular}

\section{Encamisado con ángulos y soleras de acero}

Se comienza con las columnas con mayor probabilidad de falla de ambas escuelas. A continuación, se muestra el ejemplo de una columna que tuvo daño, se usó la guía técnica para la rehabilitación sísmica de edificios escolares de la Ciudad de México. Posteriormente, se procedió a la alternativa de refuerzo mediante el encamisado con ángulos y soleras de acero, como se ve en la figura 20. A la sección se le aumentaron 4 ángulos LI $44 \mathrm{~mm} \times 8 \mathrm{~mm}$, así como soleras de $40 \mathrm{~mm}$ de ancho $\times 6 \mathrm{~mm}$ de espesor @ 15 cm. Se usó el acero A992 grado 50, se llegó a estas dimensiones para que se cumpliera con la normativa del Eurocódigo.

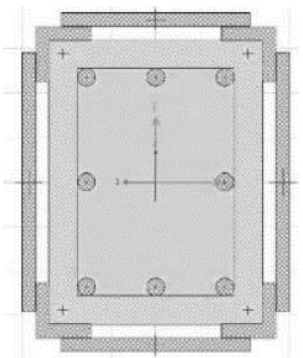

Figura 20. Columna encamisada con ángulos y soleras

Se obtuvieron los diagramas de interacción, para los 2 sentidos, M3 y M2 (figuras 21 y 22, respectivamente), de la sección compuesta, tal como se muestra a continuación.

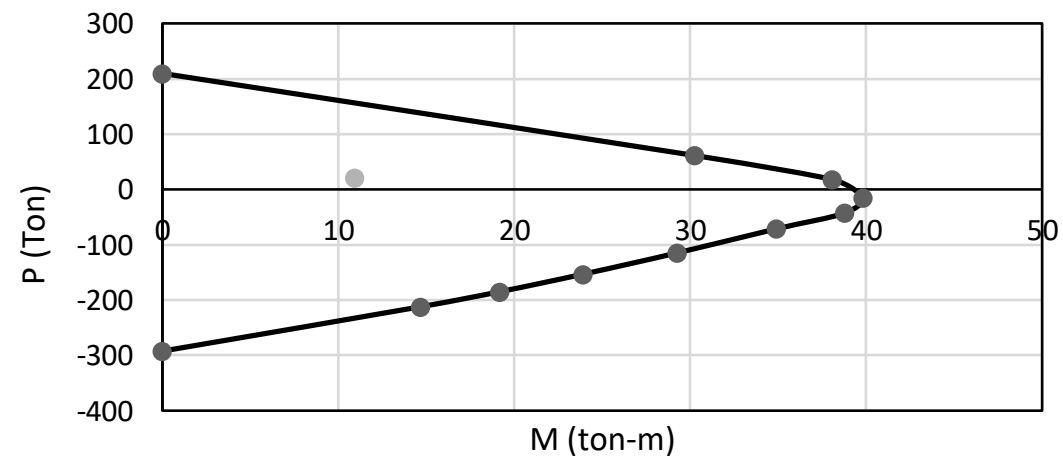

Figura 21. Diagrama interacción M3 


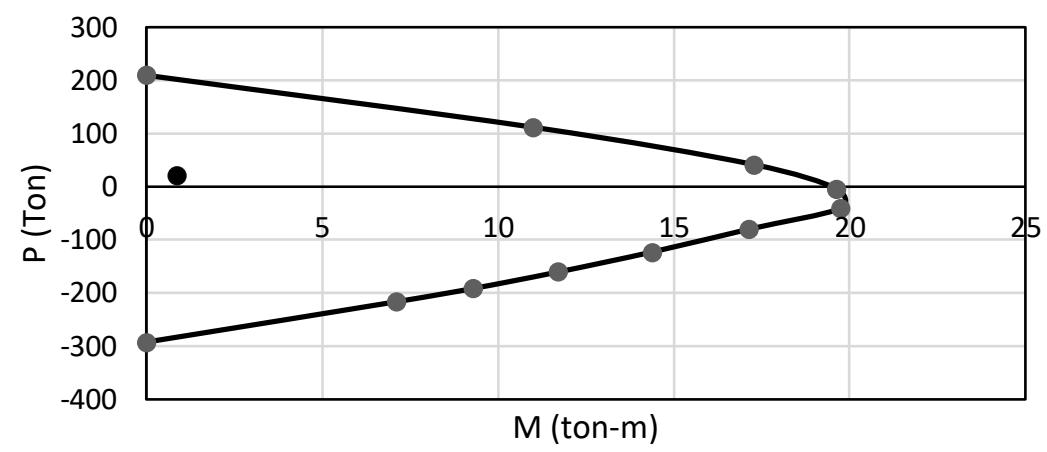

Figura 22. Diagrama interacción M2

En las tablas 11 y 12 se muestran los resultados de la alternativa de encamisado con ángulos y soleras de acero para columnas.

Tabla 11. Índices de confiabilidad de columnas (encamisado acero). Escuela Yautepec

\begin{tabular}{cccccccc}
\hline $\begin{array}{c}\text { Columna } \\
\text { Elemento \# }\end{array}$ & Actuante & \multicolumn{5}{c}{ Resistente } \\
& P (ton) & $\begin{array}{c}\text { M3 } \\
\text { (ton-m) }\end{array}$ & $\begin{array}{c}\text { M2 } \\
\text { (ton-m) }\end{array}$ & P (ton) & $\begin{array}{c}\text { M3 } \\
\text { (ton-m) }\end{array}$ & $\begin{array}{c}\text { M2 } \\
\text { (ton-m) }\end{array}$ & $\beta$ \\
\hline 1 & 18.22 & 5.17 & 0.07 & 229.25 & 23.83 & 10.89 & 4.95 \\
2 & 6.31 & 5.33 & 0.10 & 229.25 & 23.83 & 10.89 & 4.86 \\
11 & 18.24 & 5.15 & 0.08 & 229.25 & 23.83 & 10.89 & 4.96 \\
12 & 6.30 & 5.33 & 0.11 & 229.25 & 23.83 & 10.89 & 4.86 \\
17 & 21.71 & 5.18 & 0.06 & 229.25 & 23.83 & 10.89 & 4.95 \\
18 & 10.03 & 5.47 & 0.08 & 229.25 & 23.83 & 10.89 & 4.77 \\
19 & 17.08 & 5.20 & 0.09 & 229.25 & 23.83 & 10.89 & 4.93 \\
20 & 10.04 & 5.48 & 0.09 & 229.25 & 23.83 & 10.89 & 4.77 \\
25 & 21.55 & 5.19 & 0.07 & 229.25 & 23.83 & 10.89 & 4.94 \\
26 & 9.97 & 5.591 & 0.108 & 229.25 & 23.827 & 10.887 & 4.705 \\
\hline
\end{tabular}

Tabla 12. Índices de confiabilidad de columnas (encamisado acero). Escuela Tlatenchi

\begin{tabular}{cccccccc}
$\begin{array}{c}\text { Columna } \\
\text { Elemento \# }\end{array}$ & Actuante & \multicolumn{5}{c}{ Resistente } \\
& P (ton) & $\begin{array}{c}\text { M3 } \\
\text { (ton-m) }\end{array}$ & $\begin{array}{c}\text { M2 } \\
\text { (ton-m) }\end{array}$ & P (ton) & $\begin{array}{c}\text { M3 } \\
\text { (ton-m) }\end{array}$ & $\begin{array}{c}\text { M2 } \\
\text { (ton-m) }\end{array}$ & $\beta$ \\
\hline 3 & 5.13 & 10.91 & 0.78 & 292.25 & 39.00 & 19.44 & 4.15 \\
4 & 1.67 & 8.86 & 0.24 & 229.25 & 23.83 & 10.89 & 3.85 \\
5 & 5.12 & 10.91 & 0.50 & 292.25 & 39.00 & 19.44 & 4.15 \\
6 & 1.67 & 8.83 & 0.75 & 229.25 & 23.83 & 10.89 & 3.86 \\
25 & 20.27 & 10.91 & 0.88 & 292.25 & 39.00 & 19.44 & 4.15 \\
26 & 9.22 & 8.61 & 0.32 & 229.25 & 23.83 & 10.89 & 3.94 \\
27 & 16.06 & 10.91 & 0.66 & 292.25 & 39.00 & 19.44 & 4.15 \\
28 & 9.39 & 8.57 & 0.90 & 229.25 & 23.83 & 10.89 & 3.96 \\
33 & 4.923 & 10.513 & 0.867 & 292.25 & 39.003 & 19.44 & 4.266 \\
\hline
\end{tabular}

En la figura 23 se muestra un ejemplo del encamisado de trabes (Alcocer y otros, 2019). 

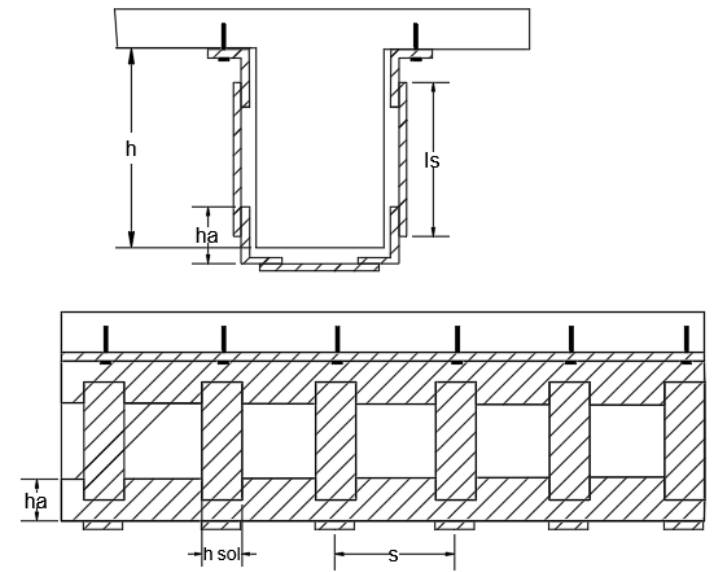

Figura 23. Trabe encamisada con ángulos y soleras

Después de consultar la guía técnica, se muestra a continuación el ejemplo de una columna dañada. Se llegó a las dimensiones y refuerzo del acero mostradas en la figura 24.
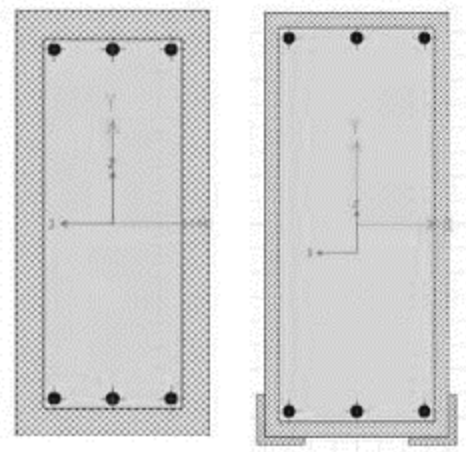

Figura 24. Encamisado de trabe

En las tablas 13 y 14 se muestran los índices de confiabilidad de la alternativa de refuerzo con perfiles de acero para trabes. El cálculo sigue los pasos mencionados para la alternativa 1.

Tabla 13. Índices de confiabilidad de trabes (alternativa acero). Escuela Yautepec

\begin{tabular}{|c|c|c|c|c|c|c|c|c|c|}
\hline \multirow{2}{*}{$\begin{array}{c}\text { Trabe } \\
\text { Elemento \# }\end{array}$} & \multicolumn{2}{|c|}{ Actuante } & \multicolumn{2}{|c|}{ Resistente } & \multirow{2}{*}{$\begin{array}{l}\text { Mact/ } \\
\text { M res }\end{array}$} & \multirow{2}{*}{$\begin{array}{l}\text { Vact/ } \\
\text { V res }\end{array}$} & \multirow{2}{*}{$\begin{array}{c}\text { Mediana } \\
\text { Ma }\end{array}$} & \multirow{2}{*}{$\begin{array}{c}\text { Mediana } \\
\mathrm{Mr}\end{array}$} & \multirow{2}{*}{$\beta$} \\
\hline & $\begin{array}{c}\mathrm{M} \\
\text { (ton-m) }\end{array}$ & $\begin{array}{c}\mathrm{V} \\
\text { (ton) }\end{array}$ & $\begin{array}{c}\mathrm{M} \\
\text { (ton-m) }\end{array}$ & $\begin{array}{c}\mathrm{V} \\
\text { (ton) }\end{array}$ & & & & & \\
\hline 23 & 8.64 & 5.65 & 33.46 & 28.65 & 0.26 & 0.2 & 7.93 & 33.29 & 4.54 \\
\hline 24 & 6.42 & 5.17 & 18.75 & 18.99 & 0.34 & 0.27 & 5.89 & 18.66 & 3.85 \\
\hline 31 & 8.69 & 5.65 & 33.46 & 28.65 & 0.26 & 0.2 & 7.97 & 33.29 & 4.52 \\
\hline 32 & 6.43 & 5.18 & 18.75 & 18.99 & 0.34 & 0.27 & 5.9 & 18.66 & 3.84 \\
\hline 39 & 8.69 & 5.65 & 33.46 & 28.65 & 0.26 & 0.2 & 7.97 & 33.29 & 4.52 \\
\hline 40 & 6.44 & 5.18 & 18.75 & 18.99 & 0.34 & 0.27 & 5.9 & 18.66 & 3.84 \\
\hline 47 & 8.77 & 5.63 & 33.46 & 28.65 & 0.26 & 0.2 & 8.05 & 33.29 & 4.49 \\
\hline 48 & 6.51 & 5.18 & 18.75 & 18.99 & 0.35 & 0.27 & 5.98 & 18.66 & 3.8 \\
\hline 55 & 8.66 & 2.34 & 33.46 & 28.65 & 0.26 & 0.08 & 7.94 & 33.29 & 4.53 \\
\hline 56 & 5.58 & 2.59 & 18.75 & 18.99 & 0.3 & 0.14 & 5.12 & 18.66 & 4.09 \\
\hline
\end{tabular}


Tabla 14. Índices de confiabilidad de trabes (alternativa acero). Escuela Tlatenchi

\begin{tabular}{cccccccccc}
\hline $\begin{array}{c}\text { Trabe } \\
\text { Elemento \# }\end{array}$ & $\begin{array}{c}\mathrm{M} \\
\text { (ton-m) }\end{array}$ & $\begin{array}{c}\mathrm{V} \\
\text { (ton) }\end{array}$ & $\begin{array}{c}\text { Resistente } \\
\text { (ton-m) }\end{array}$ & $\begin{array}{c}\mathrm{V} \\
\text { (ton) }\end{array}$ & $\begin{array}{c}\text { Mact / } \\
\text { M res }\end{array}$ & $\begin{array}{c}\text { Vact / } \\
\text { V res }\end{array}$ & $\begin{array}{c}\text { Mediana } \\
\text { Ma }\end{array}$ & $\begin{array}{c}\text { Mediana } \\
\text { Mr }\end{array}$ & $\beta$ \\
\hline 9 & 10.46 & 2.68 & 36.66 & 28.65 & 0.004 & 0.005 & 9.60 & 36.48 & 4.22 \\
10 & 8.79 & 2.58 & 36.66 & 28.65 & 0.005 & 0.005 & 8.07 & 36.48 & 4.77 \\
31 & 10.46 & 5.67 & 36.66 & 28.65 & 0.009 & 0.011 & 9.60 & 36.48 & 4.22 \\
32 & 8.56 & 5.19 & 36.66 & 28.65 & 0.010 & 0.010 & 7.85 & 36.48 & 4.86 \\
39 & 10.46 & 5.65 & 36.66 & 28.65 & 0.009 & 0.011 & 9.60 & 36.48 & 4.22 \\
40 & 8.45 & 5.19 & 36.66 & 28.65 & 0.010 & 0.010 & 7.76 & 36.48 & 4.90 \\
47 & 10.46 & 5.66 & 36.66 & 28.65 & 0.009 & 0.010 & 9.60 & 36.48 & 4.22 \\
48 & 8.26 & 5.20 & 36.66 & 28.65 & 0.010 & 0.010 & 7.58 & 36.48 & 4.97 \\
55 & 10.46 & 2.66 & 36.66 & 28.65 & 0.004 & 0.004 & 9.60 & 36.48 & 4.22 \\
56 & 8.16 & 2.60 & 36.66 & 28.65 & 0.006 & 0.005 & 7.49 & 36.48 & 5.01 \\
63 & 10.46 & 2.65 & 36.66 & 28.65 & 0.004 & 0.004 & 9.59 & 36.48 & 4.22 \\
\hline
\end{tabular}

\section{Muros de concreto}

Se comienza con la demolición de muros de mampostería existentes, y se introducen muros de concreto reforzado de 3000 psi o $\mathrm{f}^{\prime} \mathrm{c}=210 \mathrm{~kg} / \mathrm{cm}^{2}$ y un módulo de elasticidad de $\mathrm{E}=219,499 \mathrm{~kg} / \mathrm{cm}^{2}$ con acero de refuerzo del \#3, @ $20 \mathrm{~cm}$, en ambas caras (figura 25).

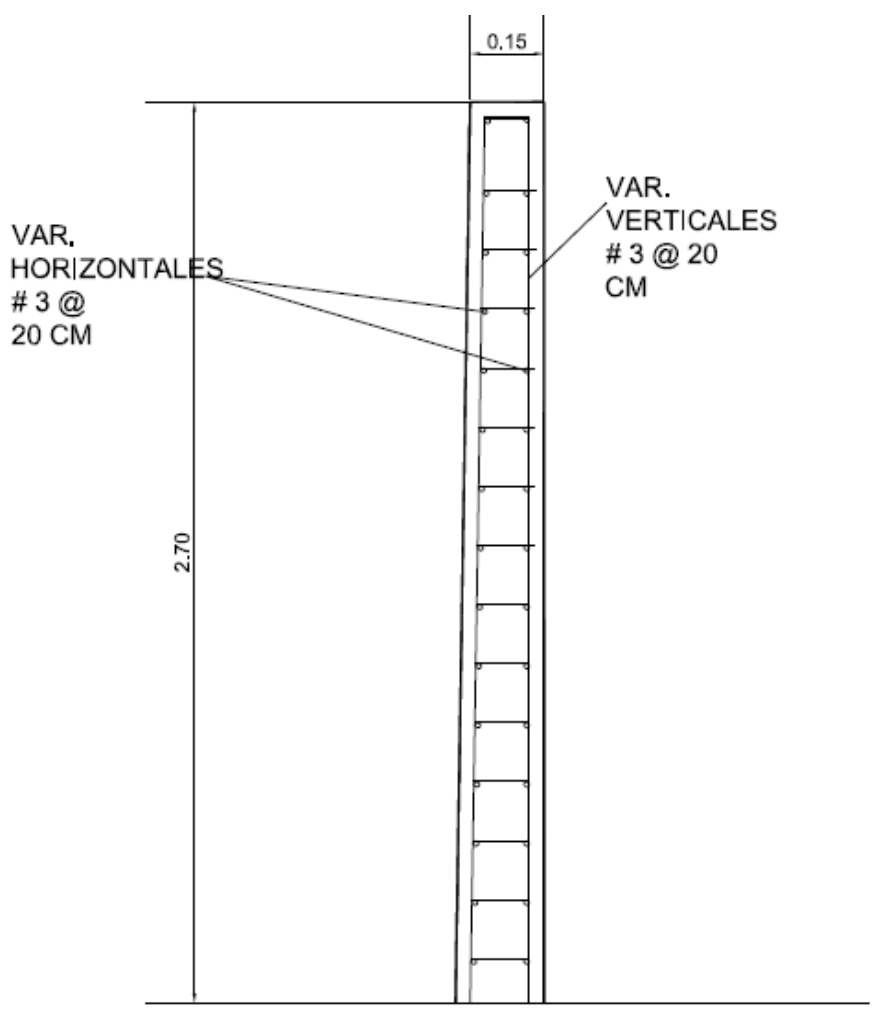

Figura 25. Armado muro concreto

En las figuras 26, 27, 28 y 29 se muestra en que parte se colocaron muros de concreto de $15 \mathrm{~cm}$ de espesor (color negro) y los muros de mampostería de $12 \mathrm{~cm}$ (líneas punteadas), para ambas escuelas. 


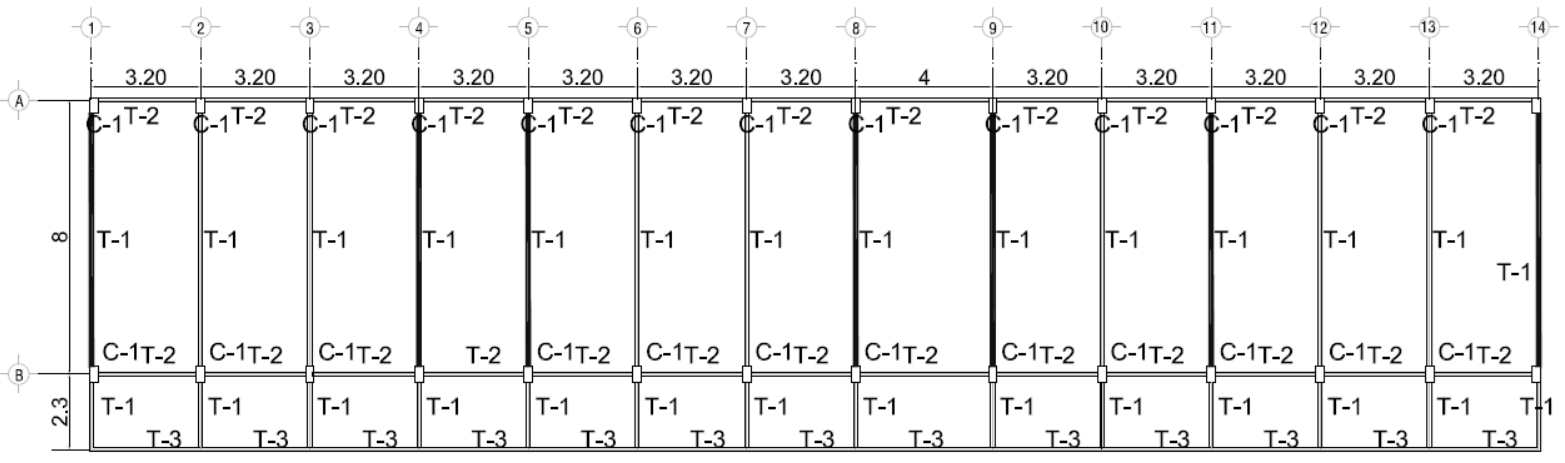

Figura 26. Ubicación de muros de concreto. Yautepec, planta baja

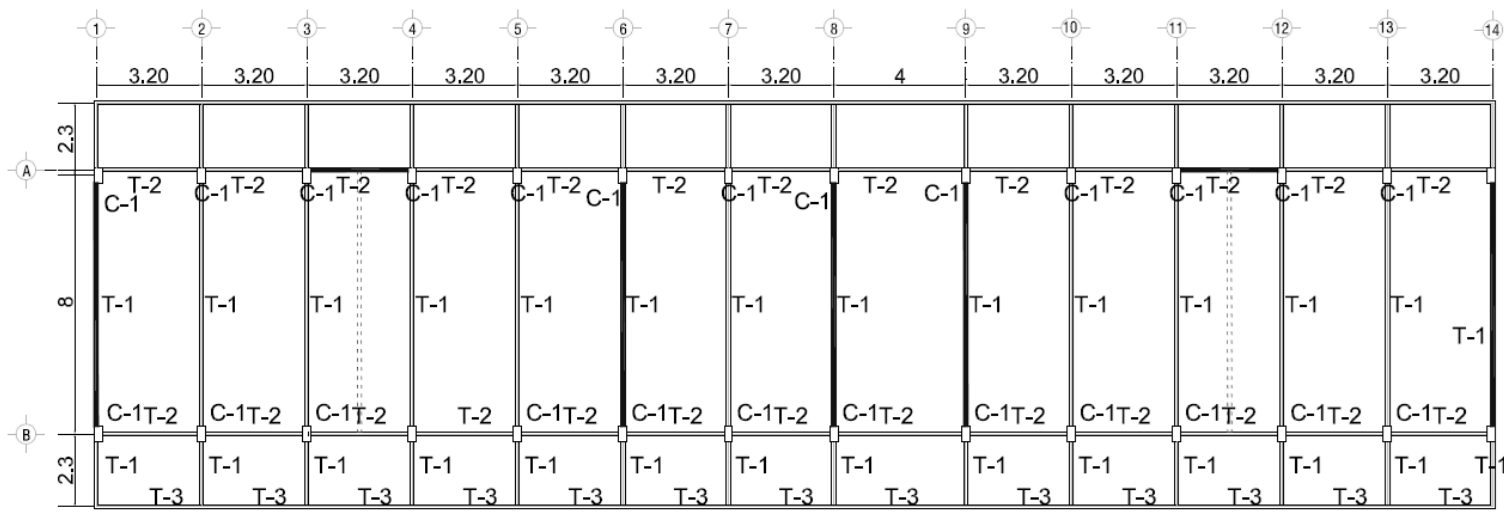

Figura 27. Ubicación de muros de concreto. Yautepec, primer nivel

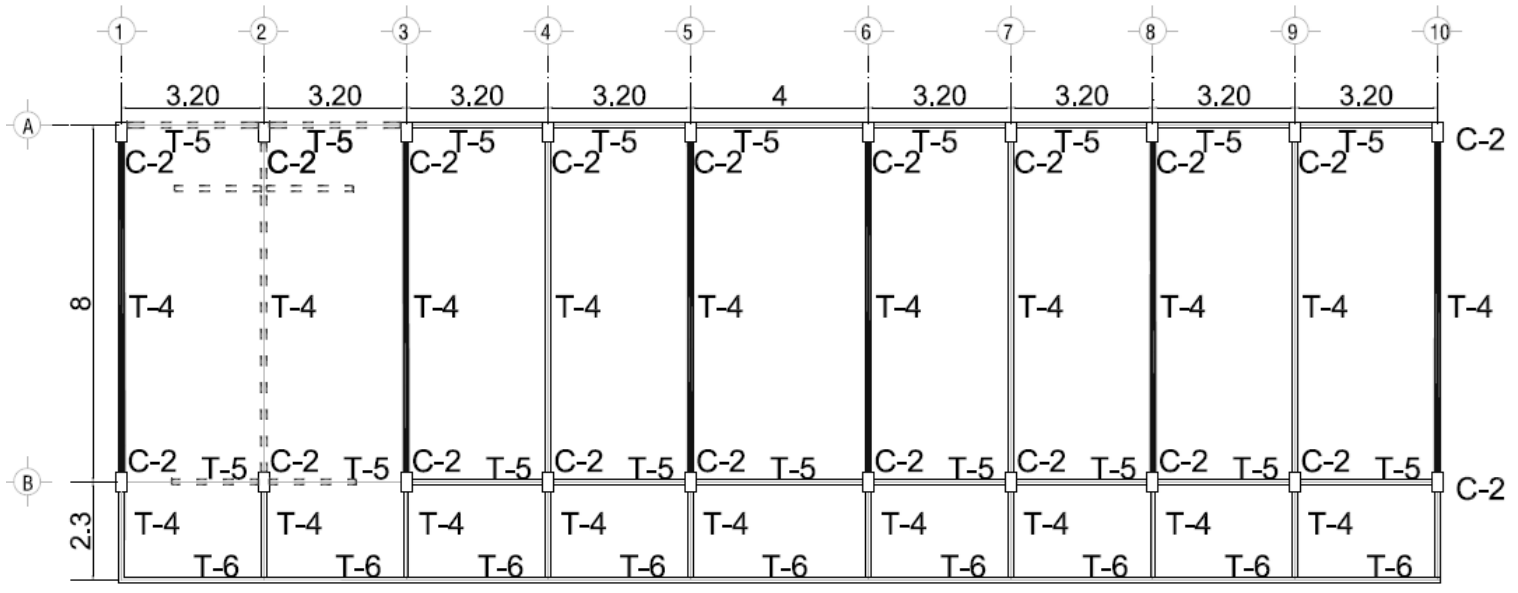

Figura 28. Ubicación de muros de concreto. Tlatenchi, planta baja 


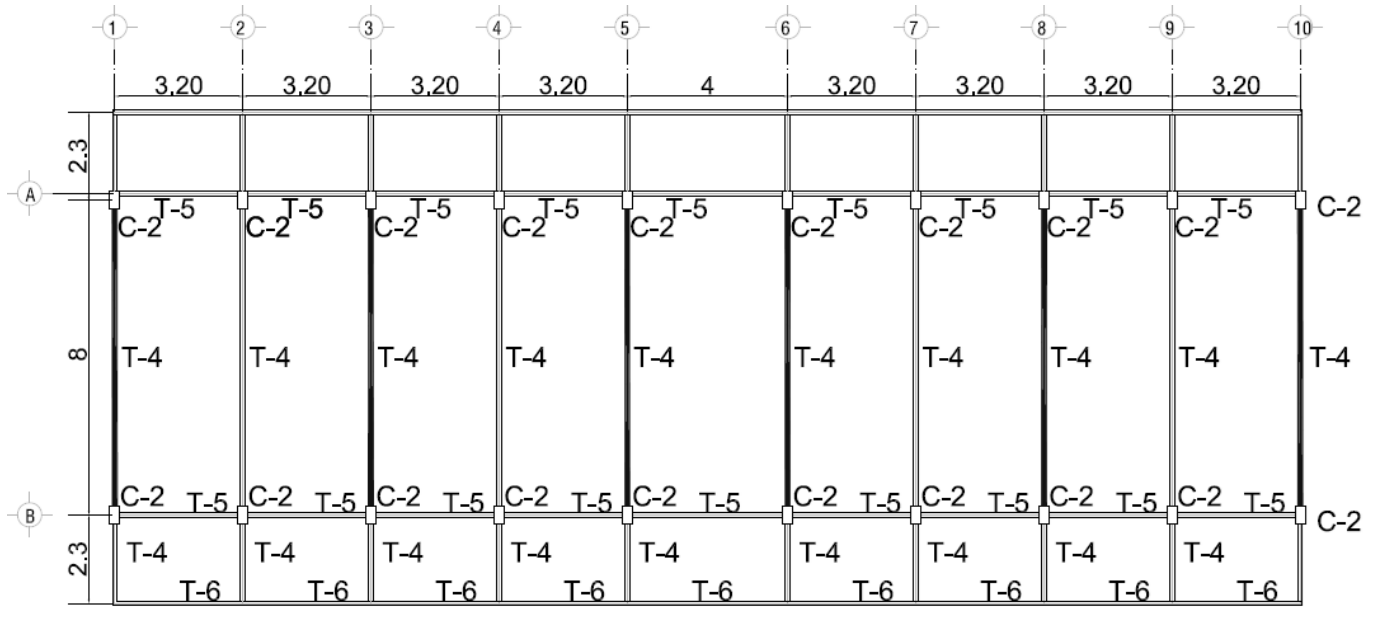

Figura 29. Ubicación de muros de concreto. Tlatenchi, primer nivel

En las tablas 15, 16, 17 y 18 se muestran los resultados arrojados por esta alternativa, para columnas y trabes.

Tabla 15. Índices de confiabilidad de columnas (muro concreto $15 \mathrm{~cm}$ ). Escuela Yautepec

\begin{tabular}{cccccccc}
\hline & \multicolumn{3}{c}{ Actuante } & \multicolumn{3}{c}{ Resistente } \\
Columna \# & P (ton) & $\begin{array}{c}\text { M3 } \\
\text { (ton-m) }\end{array}$ & $\begin{array}{c}\text { M2 } \\
\text { (ton-m) }\end{array}$ & P (ton) & $\begin{array}{c}\text { M2 } \\
\text { (ton-m) }\end{array}$ & $\beta$ \\
& ton-m) & \\
\hline 1 & 18.78 & 0.20 & 0.07 & 229.25 & 23.83 & 10.89 & 15.26 \\
2 & 5.59 & 0.16 & 0.15 & 229.25 & 23.83 & 10.89 & 15.92 \\
11 & 14.42 & 0.18 & 0.06 & 229.25 & 23.83 & 10.89 & 15.61 \\
12 & 4.50 & 0.14 & 0.12 & 229.25 & 23.83 & 10.89 & 16.31 \\
17 & 21.25 & 0.20 & 0.06 & 229.25 & 23.83 & 10.89 & 15.17 \\
18 & 9.80 & 0.20 & 0.10 & 229.25 & 23.83 & 10.89 & 15.19 \\
19 & 16.54 & 0.24 & 0.04 & 229.25 & 23.83 & 10.89 & 14.72 \\
20 & 9.68 & 0.23 & 0.07 & 229.25 & 23.83 & 10.89 & 14.83 \\
25 & 20.82 & 0.22 & 0.04 & 229.25 & 23.83 & 10.89 & 14.99 \\
26 & 9.63 & 0.26 & 0.06 & 229.25 & 23.83 & 10.89 & 14.45 \\
\hline
\end{tabular}

Tabla 16. Índices de confiabilidad de columnas (muro concreto $15 \mathrm{~cm}$ ). Escuela Tlatenchi

\begin{tabular}{|c|c|c|c|c|c|c|c|}
\hline \multirow{2}{*}{$\begin{array}{c}\text { Columna } \\
\text { Elemento \# }\end{array}$} & \multicolumn{3}{|c|}{ Actuante } & \multicolumn{3}{|c|}{ Resistente } & \multirow{2}{*}{$\beta$} \\
\hline & $\mathrm{P}$ (ton) & $\begin{array}{c}\text { M3 } \\
\text { (ton-m) }\end{array}$ & $\begin{array}{c}\text { M2 } \\
\text { (ton-m) }\end{array}$ & $\mathrm{P}$ (ton) & $\begin{array}{c}\text { M3 } \\
\text { (ton-m) }\end{array}$ & $\begin{array}{c}\text { M2 } \\
\text { (ton-m) }\end{array}$ & \\
\hline 3 & 18.68 & 0.36 & 0.08 & 229.25 & 23.83 & 10.89 & 13.37 \\
\hline 4 & 4.82 & 0.15 & 0.16 & 229.25 & 23.83 & 10.89 & 16.09 \\
\hline 5 & 21.36 & 0.34 & 0.08 & 229.25 & 23.83 & 10.89 & 13.56 \\
\hline 6 & 6.16 & 0.14 & 0.17 & 229.25 & 23.83 & 10.89 & 16.46 \\
\hline 25 & 20.94 & 0.37 & 0.05 & 229.25 & 23.83 & 10.89 & 13.28 \\
\hline 26 & 9.64 & 0.21 & 0.09 & 229.25 & 23.83 & 10.89 & 15.16 \\
\hline 27 & 16.62 & 0.40 & 0.06 & 229.25 & 23.83 & 10.89 & 13.04 \\
\hline 28 & 9.75 & 0.23 & 0.10 & 229.25 & 23.83 & 10.89 & 14.81 \\
\hline 33 & 20.06 & 0.41 & 0.00 & 229.25 & 23.83 & 10.89 & 13.01 \\
\hline 34 & 9.276 & 0.282 & 0.003 & 229.25 & 23.827 & 10.887 & 14.153 \\
\hline
\end{tabular}


Tabla 17. Índices de confiabilidad de trabes (muros de concreto). Escuela Yautepec

\begin{tabular}{|c|c|c|c|c|c|c|c|c|c|}
\hline \multirow[b]{2}{*}{$\begin{array}{c}\text { Trabe } \\
\text { Elemento \# }\end{array}$} & \multicolumn{2}{|c|}{ Actuante } & \multicolumn{2}{|c|}{ Resistente } & \multirow[b]{2}{*}{$\begin{array}{l}\text { Mact/ } \\
\text { M res }\end{array}$} & \multirow[b]{2}{*}{$\begin{array}{l}\text { Vact/ } \\
\text { V res }\end{array}$} & \multirow[b]{2}{*}{$\begin{array}{c}\text { Mediana } \\
\text { Ma }\end{array}$} & \multirow[b]{2}{*}{$\begin{array}{c}\text { Mediana } \\
\text { Mr }\end{array}$} & \multirow[b]{2}{*}{$\beta$} \\
\hline & $\begin{array}{c}\mathrm{M} \\
\text { (ton-m) }\end{array}$ & $\begin{array}{c}\mathrm{V} \\
\text { (ton) }\end{array}$ & $\begin{array}{c}\mathrm{M} \\
\text { (ton-m) }\end{array}$ & $\begin{array}{c}\mathrm{V} \\
\text { (ton) }\end{array}$ & & & & & \\
\hline 23 & 6.51 & 5.72 & 18.75 & 18.99 & 0.35 & 0.3 & 5.98 & 18.66 & 3.9 \\
\hline 24 & 6.58 & 5.22 & 18.75 & 18.99 & 0.35 & 0.27 & 6.04 & 18.66 & 3.87 \\
\hline 31 & 6.67 & 5.75 & 18.75 & 18.99 & 0.36 & 0.3 & 6.12 & 18.66 & 3.82 \\
\hline 32 & 6.84 & 5.27 & 18.75 & 18.99 & 0.36 & 0.28 & 6.27 & 18.66 & 3.75 \\
\hline 39 & 6.6 & 5.73 & 18.75 & 18.99 & 0.35 & 0.3 & 6.06 & 18.66 & 3.86 \\
\hline 40 & 6.79 & 5.25 & 18.75 & 18.99 & 0.36 & 0.28 & 6.23 & 18.66 & 3.87 \\
\hline 47 & 6.47 & 5.71 & 18.75 & 18.99 & 0.35 & 0.3 & 5.94 & 18.66 & 3.2 \\
\hline 48 & 6.68 & 5.22 & 18.75 & 18.99 & 0.36 & 0.28 & 6.13 & 18.66 & 3.82 \\
\hline 55 & 2.5 & 2.4 & 18.75 & 18.99 & 0.13 & 0.13 & 2.29 & 18.66 & 6.63 \\
\hline 56 & 3.8 & 2.63 & 18.75 & 18.99 & 0.2 & 0.14 & 3.48 & 18.66 & 5.31 \\
\hline
\end{tabular}

Tabla 18. Índices de confiabilidad de trabes (muros de concreto). Escuela Tlatenchi

\begin{tabular}{cccccccccc}
\hline $\begin{array}{c}\text { Trabe } \\
\text { Elemento \# }\end{array}$ & \multicolumn{2}{c}{ Actuante } & \multicolumn{2}{c}{ Resistente } & Mact/ & Vact/ & Mediana \\
Ma & V & M & V & M res & V res & Mrana & $\beta$ \\
& (ton-m) & (ton) & (ton-m) & (ton) & & & & & \\
\hline 9 & 2.82 & 2.5 & 18.75 & 18.99 & 0.15 & 0.13 & 2.58 & 18.66 & 6.25 \\
10 & 3.26 & 2.61 & 18.75 & 18.99 & 0.17 & 0.14 & 2.99 & 18.66 & 5.79 \\
31 & 6.51 & 5.72 & 18.75 & 18.99 & 0.35 & 0.3 & 5.98 & 18.66 & 3.85 \\
32 & 6.59 & 5.22 & 18.75 & 18.99 & 0.35 & 0.27 & 6.04 & 18.66 & 3.56 \\
39 & 6.48 & 5.71 & 18.75 & 18.99 & 0.35 & 0.3 & 5.95 & 18.66 & 3.62 \\
40 & 6.63 & 5.22 & 18.75 & 18.99 & 0.35 & 0.27 & 6.08 & 18.66 & 3.8 \\
47 & 6.46 & 5.71 & 18.75 & 18.99 & 0.34 & 0.3 & 5.93 & 18.66 & 3.62 \\
48 & 6.7 & 5.22 & 18.75 & 18.99 & 0.36 & 0.28 & 6.14 & 18.66 & 3.8 \\
55 & 2.5 & 2.4 & 18.75 & 18.99 & 0.13 & 0.13 & 2.3 & 18.66 & 6.62 \\
56 & 3.8 & 2.63 & 18.75 & 18.99 & 0.2 & 0.14 & 3.49 & 18.66 & 5.81 \\
\hline
\end{tabular}

Después de tener los índices de confiabilidad para todas las alternativas se procede al cálculo de la probabilidad de falla global (PFG) de cada alternativa. Este proceso consiste en identificar el marco más crítico, es decir las 2 columnas (F1 y F2) y la trabe (F3) que tienen las probabilidades de falla locales mayores, se utilizan las expresiones $6,7,8$ y 9 , y finalmente se sustituyen en la expresión 5. Los resultados se resumen en las tablas $19,19.1,20$ y 20.1.

Tabla 19. Probabilidad de falla global de la escuela Yautepec en condición original

\begin{tabular}{ccc} 
Elemento \# & Condición original & PFG \\
\hline $\mathrm{P}\left(\mathrm{F}_{1}\right)$ & 0.00000596 & \\
$\mathrm{P}\left(\mathrm{F}_{2}\right)$ & 0.00000588 & 0.0115 \\
$\mathrm{P}\left(\mathrm{F}_{3}\right)$ & 0.00575 & \\
\hline
\end{tabular}


Tabla 19.1. Probabilidades de falla globales de la escuela Yautepec con las 3 alternativas de reparación

\begin{tabular}{ccccccc}
\hline $\mathrm{P}(\mathrm{Fn})$ & $\begin{array}{c}\text { Enc. } \\
\text { Concreto }\end{array}$ & PFG & Enc. Acero & PFG & $\begin{array}{c}\text { Muro } \\
\text { concreto 15 } \\
\text { cm espesor }\end{array}$ & PFG \\
\hline $\mathrm{P}\left(\mathrm{F}_{1}\right)$ & 0.00000596 & & 0.00000596 & & & \\
$\mathrm{P}\left(\mathrm{F}_{2}\right)$ & 0.00000588 & $2.3 \times 10^{-4}$ & 0.00000588 & $1.90 \times 10^{-5}$ & 0 & $3.16 \times 10^{-4}$ \\
$\mathrm{P}\left(\mathrm{F}_{3}\right)$ & 0.000112 & & 0.00000654 & & & \\
\hline
\end{tabular}

Tabla 20. Probabilidad de falla global de la escuela Tlatenchi en condición original

\begin{tabular}{ccc}
$\mathrm{P}\left(\mathrm{F}_{\mathrm{n}}\right)$ & $\begin{array}{c}\text { Condición } \\
\text { original }\end{array}$ & $\mathrm{PFG}$ \\
\hline $\mathrm{P}\left(\mathrm{F}_{1}\right)$ & $4.78 \times 10^{-4}$ & \\
$\mathrm{P}\left(\mathrm{F}_{2}\right)$ & $4.78 \times 10^{-3}$ & 0.0403 \\
$\mathrm{P}\left(\mathrm{F}_{3}\right)$ & $1.78 \times 10^{-2}$ & \\
\hline
\end{tabular}

Tabla 20.1. Probabilidades de falla globales de la escuela Tlatenchi con las 3 alternativas de reparación

\begin{tabular}{ccccccc}
\hline Elemento\# & $\begin{array}{c}\text { Enc. } \\
\text { concreto }\end{array}$ & PFG & Enc. acero & PFG & $\begin{array}{c}\text { Muro } \\
\text { concreto } \\
15 \mathrm{~cm} \\
\text { espesor }\end{array}$ & PFG \\
\hline $\mathrm{P}\left(\mathrm{F}_{1}\right)$ & $8.13 \times 10^{-4}$ & & $8.13 \times 10^{-4}$ & 0 & \\
$\mathrm{P}\left(\mathrm{F}_{2}\right)$ & $8.73 \times 10^{-4}$ & $3.31 \times 10^{-3}$ & $8.73 \times 10^{-4}$ & $3.31 \times 10^{-3}$ & 0 & $4.44 \times 10^{-3}$ \\
$\mathrm{P}\left(\mathrm{F}_{3}\right)$ & $1.22 \times 10^{-3}$ & & $1.22 \times 10^{-3}$ & & $2.22 \times 10^{-4}$ & \\
\hline
\end{tabular}

\section{Costo de reparación y daños}

Se procede a calcular los costos de las reparaciones de cada alternativa así, como las consecuencias económicas, haciendo uso de las ecuaciones 10,11, 12 y 13. Los resultados se resumen en la tabla 21. El factor de valor presente se calcula en términos de la tasa de interés neta que es, 9\%, y la vida útil de la escuela, que es de 50 años. Se observa en la Tabla 21, que el costo por pérdida de vidas es el más crítico. Estos costos se obtienen como se describió en la sección de cálculo de costos esperados.

Tabla 21. Costo de daños

\begin{tabular}{ccl}
\hline Tipo de costo & Yautepec & Tlatenchi \\
\hline$C_{c}$ & $\$ 233,000$ & $\$ 178,000$ \\
$C_{e}$ & $\$ 102,000$ & $\$ 142,000$ \\
$C_{i n}$ & $\$ 446,000$ & $\$ 311,000$ \\
$C_{f}$ & $\$ 317,559,000$ & $\$ 308,929,000$ \\
$C_{F}$ & $\$ 318,340,000$ & $\$ 309,560,000$ \\
\hline
\end{tabular}

Los costos esperados en el ciclo de vida se calculan con las ecuaciones 10,11 y 12 . Ver las tablas 22 y 23 para Yautepec y Tlatenchi, respectivamente, con las 3 alternativas de reparación. 
Tabla 22. Costo esperado en el ciclo de vida. Escuela de Yautepec

\begin{tabular}{ccccc}
$\begin{array}{c}\text { Alternativa } \\
\text { de } \\
\text { reparación }\end{array}$ & $\begin{array}{c}\text { Costo de } \\
\text { reparación }\end{array}$ & $P_{f}$ & $\begin{array}{c}\text { Costo futuro sin el } \\
\text { costo de reparación }\end{array}$ & $\begin{array}{c}\text { Costo esperado en el } \\
\text { ciclo de vida }\end{array}$ \\
\hline Concreto & $\$ 342,000$ & $2.30 \times 10^{-4}$ & $\$ 805,000$ & $\$ 1,148,000$ \\
Acero & $\$ 133,000$ & $1.90 \times 10^{-5}$ & $\$ 66,000$ & $\$ 199,000$ \\
Muros & $\$ 555,000$ & $3.16 \times 10^{-4}$ & $\$ 1,106,000$ & $\$ 1,662,000$ \\
\hline
\end{tabular}

Tabla 23. Costo esperado en el ciclo de vida. Escuela de Tlatenchi

\begin{tabular}{ccccc}
\hline $\begin{array}{c}\text { Alternativa } \\
\text { de } \\
\text { reparación }\end{array}$ & Costo de reparación & $P_{f}$ & $\begin{array}{c}\text { Costo futuro sin el } \\
\text { costo de reparación }\end{array}$ & $\begin{array}{c}\text { Costo esperado en } \\
\text { el ciclo de vida }\end{array}$ \\
\hline Concreto & $\$ 923,000$ & $3.31 \times 10^{-3}$ & $\$ 1,250,000$ & $\$ 12,173,000$ \\
Acero & $\$ 601,000$ & $3.31 \times 10^{-3}$ & $\$ 1,250,000$ & $\$ 11,852,000$ \\
Muros & $\$ 590,000$ & $4.44 \times 10^{-4}$ & $\$ 1,510,000$ & $\$ 2,101,000$ \\
\hline
\end{tabular}

\section{ANÁLISIS DE RESULTADOS}

La opción óptima de reparación, de las 3 consideradas, es la que presente la menor relación costobeneficio, siendo el beneficio el incremento en confiabilidad o reducción de probabilidad de falla y el costo es el esperado en el ciclo de vida. Dicha relación se calcula como el costo esperado en el ciclo de vida, y la opción que presente el menor costo esperado se elige como óptima. Como se observa en las tablas 22 y 23 , la reparación óptima para la escuela Yautepec es, la opción de reparación 2, con ángulos y soleras de acero, ya que el costo esperado en el ciclo de vida fue el menor de los otros 2 costos de reparación con solo $\$ 199,000$ pesos mexicanos para su costo esperado en su ciclo de vida. Para Tlatenchi, la reparación óptima es colocación de muros de concreto de $15 \mathrm{~cm}$ de espesor con $\$ 2^{\prime} 101,000$ pesos mexicanos para su costo esperado en su ciclo de vida. En la tabla 24 se muestran los valores mínimos recomendados por el Eurocódigo. (The European Union Per Regulation, 2002).

Tabla 24. Valores mínimos recomendados para el índice de confiabilidad $\beta$

\begin{tabular}{ccc}
\hline & \multicolumn{2}{c}{ Valores mínimos para $\beta$} \\
$\begin{array}{c}\text { Clase de } \\
\text { confiabilidad } \\
\text { referencia 1 } \\
\text { año }\end{array}$ & $\begin{array}{c}\text { Periodo de } \\
\text { referencia 50 } \\
\text { años }\end{array}$ \\
\hline RC3 & 5.2 & 4.3 \\
RC2 & 4.7 & 3.8 \\
RC1 & 4.2 & 3.3 \\
\hline
\end{tabular}

En el caso de ambas escuelas según el Eurocódigo 1990, según la clase de consecuencia en CC2, se menciona que, para elementos estructurales individuales, para un periodo de referencia de 50 años el índice de confiabilidad debe ser mínimo $\beta=3.8$, lo cual cumple en todos los elementos. 
El costo por pérdida de vidas es el más crítico, dado que existe un gran número de estudiantes cuya vida, desafortunadamente, se perdería. Para cada alternativa y para cada escuela, se consideró que un nivel de confiabilidad satisfactorio es cuando el índice de confiabilidad es mayor que 3.8 para el caso de las escuelas analizadas, pero no muy por encima de ese valor para evitar ser excesivamente conservador y costoso. Para cada opción de reparación se probaron varios niveles de reparación, es decir, dimensiones de secciones, perfiles de acero, áreas de varillas de acero, número de varillas, hasta alcanzar el nivel de confiabilidad adecuado. En lo que respecta a la evaluación de costos de reparación, se incluyen materiales y costos laborales y, para las posibles consecuencias futuras de fallas, se consideran los costos actuales en México y se siguieron las prácticas típicas para los tipos de reparación, la duración de la reparación, la sustitución de instalaciones y las políticas académicas.

\section{CONCLUSIONES Y RECOMENDACIONES}

Se usó una formulación basada en riesgo, para evaluar alternativas de refuerzo para escuelas en zonas sísmicas y seleccionar la alternativa óptima. Se calibró el procedimiento mediante la comparación del desempeño estructural ante demandas sísmicas del sismo de 2017, con los daños observados en las escuelas. De los resultados obtenidos, para las opciones de reparación examinadas no se puede generalizar que una técnica de reparación sea superior a otra, depende de cada caso en particular. Cabe mencionar que con perfiles de acero es más fácil llegar al resultado deseado y no ser tan conservador, ya que se puede ir iterando diferentes perfiles, con diferentes espesores. No es así con concreto, ya que como menciona la guía técnica, los recubrimientos y acero mínimos para los encamisados, puede llevar en algunas ocasiones a resultados muy conservadores.

La opción óptima depende de las áreas de planta, elevaciones, el acero de refuerzo que tienen originalmente, el tamaño de los elementos estructurales y el peligro sísmico del sitio. Por ello para cada escuela va a ser diferente la opción de reparación óptima, no siempre va a ser la misma. En los casos estudiados se tienen 2 escuelas de 2 niveles cada una, pero con diferentes dimensiones en planta. Para la primera escuela la reparación óptima fue con perfiles de acero que cambio la probabilidad de falla global de $0.0115 \times 10^{-5}$ a $1.90 \times 10^{-5}$, para la segunda escuela fue con la introducción de muros de concreto de $15 \mathrm{~cm}$ de espesor, llevando de una probabilidad de falla global de $0.0403 \times 10^{-3}$ a $4.44 \times 10^{-3}$.

De manera preliminar, y a reserva de complementar el estudio con más casos, se observa que, si los daños son generalizados, conviene reparar todo el sistema estructural, incluyendo la adición de muros. Pero, si los daños son muy locales, conviene reparar con encamisados de concreto y/o acero.

Se recomienda generalizar este tipo de estudios para considerar otras escuelas localizadas en sitios de distinto peligro sísmico, con distintas áreas de construcción y número de niveles, para desarrollar recomendaciones más generales aplicables a escuelas de todo el país.

Se deben considerar otras combinaciones de sistemas estructurales, tamaño escolar, materiales de construcción y nivel de daños observados para establecer una base más amplia para actualizar el actual código de diseño sísmico y reparación de estructuras en México.

\section{REFERENCIAS}

Alcocer, S, D Muriá, J Abarca, R Bautista, G Bogoya, V Cruz (2020) "Assessment of the seismic safety of school buildings in Mexico: A first look". Earthquake Spectra, 36 (2_suppl); DOI: 10.1177\%2F8755293020926184 
Alcocer, S, D Muriá, J Abarca, R Bautista, G Bogoya, V Cruz, Y Martínez, B Moctezuma, D Ramírez, y G Valencia (2019), Guía técnica para la rehabilitación sísmica de edificios escolares de la Ciudad de México, Instituto de Ingeniería UNAM, Páginas 69-85, México. https://www.ilife.cdmx.gob.mx/storage/app/uploads/public/5e6/6a9/0d0/5e66a90d0c3fd540339007. pdf

American Society of Civil Engineers (ASCE/SEI 41-13, 2019), Seismic evaluation and retrofit of existing buildings, Page 255, Virginia, USA. DOI: 10.1061/9780784412855

Ang, A H-S y D De León (1997), "Determination of optimal target reliabilities for design and upgrading of structures”, Structural Safety, Part of special issue: Asian-Pacific Symposium on Structural Reliability and Its Application, Volume 19, Pages 91-103. DOI: 10.1016/S0167-4730(96)00029-X

Centro Nacional de Prevención de Desastres (CENAPRED) (2001), Norma para la evaluación del nivel de daño por sismo en estructuras y guía técnica de rehabilitación, Páginas 7-65, México. http://dimsec.com/wp-content/uploads/2017/10/NORMA-PARA-LA-EVALUACIO\%CC\%81NPOR-SISMO.pdf

CDS-2015 (2016), Capítulo de diseño por sismo, C.1.3, Manual de diseño de obras civiles, Comisión Federal de Electricidad, Instituto Nacional de Electricidad y Energías Limpias.

Cruz, J (2007), “Análisis de confiabilidad de edificios de concreto reforzado en zonas símicas considerando la acumulación del daño estructural durante la vida de servicio", Tesis Doctoral, División de Estudios de Posgrado, Facultad de Ingeniería, UNAM.

Cruz, V M (2013), Los Sismos. Una Amenaza Cotidiana; Instituto de Geofísica de la Universidad Nacional Autónoma de México/Centro de Instrumentación y Registro Sísmico A.C., Cires, La Caja de Cerillos Ediciones S.A. de C.V. México City, México, 2013.

De León, D y A H-S Ang (1994), “A damage model for reinforced concrete buildings. Further study with the 1985 Mexico City earthquake”, 6th ICOSSAR, Innsbruck, Austria.

De León, D y E Ismael (2020), "Optimal retrofit strategy for a school under seismic hazard including risk assessment”, Civil engineering and environmental systems. DOI: 10.1080/10286608.2021.1977798

De León, D y A Donaji (2020), “Towards a resilient design and retrofit of schools in México”, $17^{\text {th }}$ World Conference on Earthquake Engineering, Sendai, Japan.

De León, D y J L García (2021), "Reparación óptima basada en confiabilidad para una escuela con muro de México, mampostería y marcos de concreto con daños sísmicos”, XXII Congreso Nacional de ingeniería Estructural, Sala 1976-5, Aguascalientes, México.

De León, D y J L García (2021), "Cost effectiveness of retrofit alternatives for schools located on seismic zones”, Earthquakes and Structures, Techno-Press Journals, aceptado.

De León, D (1996), "Integrating socio-economics in the development of criteria for the aseismic design of reinforced concrete structures ”, Ph.D. thesis, Department of Civil and Environmental Engineering,UC Irvine, CA, USA.

Esteva, L, O Díaz, J Garcia, G Sierra y E Ismael (2002), "Life-cycle optimization in the establishment of performance-acceptance parameters for seismic design”, Structural Safety, Volume 24, pages 187204. DOI: $10.1016 / \mathrm{S} 0167-4730(02) 00024-3$.

Federal Emergency Management Agency (FEMA 445) (2006) Next-Generation performance-based seismic design guidelines, program plan for new and existing buildings. Applied Research Council for the Federal Emergency Management Agency, Report no. FEMA 445. Washington, DC. https://www.atcouncil.org/pdfs/FEMA445.pdf 
Federal Emergency Management Agency (FEMA 547) (2006) Techniques for the Seismic Rehabilitation of Existing Buildings, Federal Emergency Management Agency, Washington, USA. https://www.wbdg.org/FFC/DHS/fema547.pdf

Furlong, R W, C-T Hsu y S A Mirza (2004), “Analysis and design of concrete columns for biaxial bending”, ACI Materials Journal. 101. 413-423. DOI:10.14359/13101

García, J, G Hernández, J Corona, y U Mena (2018), "Desempeño sísmico de edificios tipo U2C-70 correspondiente a la infraestructura educativa", XXI Congreso Nacional de ingeniería Estructural, México,https://www.researchgate.net/publication/329070667.

Gilani, A S J, H K Miyamoto y T Nifuku (2018), "Seismic risk assessment and retrofit of school buildings in developing countries", Eleventh U.S. National Conference on Earthquake Engineering Integrating Science, Engineering \& Policy. Los Angeles, California.

Normas Técnicas Complementarias para Diseño y Construcción de Estructuras de Concreto (NTCConcreto) (2017), Gobierno de la Ciudad de México, diciembre.

Normas Técnicas Complementarias sobre Criterios y Acciones para el Diseño Estructural de las Edificaciones (NTC-Criterios) (2017), Gobierno de la Ciudad de México, diciembre.

Normas Técnicas Complementarias para Diseño y Construcción de Estructuras de Mampostería (NTCMampostería) (2017), Gobierno de la Ciudad de México, diciembre.

Instituto Nacional de la Infraestructura Física Educativa (INIFED) (2011), Normas y especificaciones para estudios proyectos construcción e instalaciones, Instituto Nacional de la Infraestructura Física Educativa. Volumen 4, Seguridad Estructural, tomo VI, Diseño de estructuras de acero, México.

Instituto Nacional de la Infraestructura Física Educativa (INIFED) (2021), Evaluación post sísmica de la infraestructura física educativa de México, Volumen 1 Metodología, Instituto Nacional de la Infraestructura Física Educativa. México.

Instituto Belisario Domínguez, Senado de la República (2017), El sistema escolar ante los sismos de septiembre de 2017, México. http://bibliodigitalibd.senado.gob.mx/bitstream/handle/123456789/3764/reporte_50_221117_web\% 20\%282\%29.pdf

Instituto de Ingeniería de la UNAM, Base de datos de registros acelero gráficos de la RAII-UNAM, (2021). https://aplicaciones.iingen.unam.mx/AcelerogramasRSM/Inicio.aspx

Jaimes, M A y M Niño (2017), "Cost-benefit analysis to assess seismic mitigation options in Mexican public school buildings”, Bull Earthquake Eng. Volume 15 (9), pp 3919-3942. DOI: 10.1007/s10518-017$\underline{0119-5}$.

Mansor, M N A y L Ch Siang (2019), "Structural retrofitting proposal for representative school facility", IOP Conf. Ser.: Earth Environ. Sci., 244, 012045. DOI: 10.1088/1755-1315/244/1/012045

Mlego (2021) Lego. Muebles escolares. Butacas. Recuperado el 14 de mayo de 2021, de https://mlego.com.mx

Nowak, A y K Collins (2012), Reliability of Structures. Second edition. USA: CRC Press, 2013. 391 p.

Ordaz, M y C Reyes (1999), "Earthquake hazard in Mexico City: Observations versus computations", Bulletin of the Seismological Society of America, Vol. 89, No. 5, pages 1379-1383. DOI: 10.1785/BSSA0890051379

Reinoso, E (2007), "Riesgo sísmico de la Ciudad de México". Trabajo de ingreso a la Academia de Ingeniería, México. 
Sánches, C y A Islas (2017), Recuento de los daños 7S y 19S a un mes de la tragedia, Senado de la República. Recuperado de http://bibliodigitalibd.senado.gob.mx/handle/123456789/3721

Teddy, B \& Associates (2010), Retrofitting simple buildings damaged by earthquakes, World Seismic Safety Initiative, United Nations. https://www.humanitarianlibrary.org/sites/default/files/2014/02/No.1_Manual_for_Rehabilitation_T $\underline{\text { eddys.pdf }}$

Tena, A, H Hernández, E A Godínez y L E Pérez (2021) "Mexico City during and after the September 19, 2017 earthquake: Assessment of seismic resilience and ongoing recovery process". J Civil Struct Health Monit, 11, 1275-1299. DOI: 10.1007/s13349-021-00511-X

The European Union Per Regulation (2002), “Eurocode- Basis of structural design”, pages 86-89.

Tolentino, D (2009), "Confiabilidad en edificios considerando la influencia del daño acumulado causado por sismos”, Tesis de Maestría, División de Estudios de Posgrado, Facultad de Ingeniería, UNAM.

Tolentino, D, S E Ruiz y M A Torres (2012), "Simplified closed-form expressions for the mean failure rate of structures considering structural deterioration", Structure and Infrastructure Engineering, Vol.8, No. 5, pp. 483-496. DOI: 10.1080/15732479.2010.539067 